15

\title{
Оптимизация измерений вектора силы взаимодействия в атомно-силовой микроскопии
}

\author{
(C) А.В. Анкудинов, ${ }^{1}$ А.М. Минарский ${ }^{2}$ \\ ${ }^{1}$ Физико-технический институт им. А.Ф. Иофрфе РАН, \\ 194021 Санкт-Петербург, Россия \\ ${ }^{2}$ Санкт-Петербургский национальный исследовательский Академический университет им. Ж.И. Алфёрова РАН, \\ 194021 Санкт-Петербург, Россия \\ e-mail: Alexander.Ankudinov@mail.ioffe.ru
}

Поступило в Редакцию 30 октября 2020 г.

В окончательной редакции 27 января 2021 г.

Принято к публикации 28 января 2021 г.

Рассмотрена задача оптимизации измерений трех пространственных компонент силы взаимодействия зонд-образец и соответствующего вектора смещения „идеального кантилевера“. В атомно-силовом микроскопе со схемой оптического рычага для определения этих компонент необходимо регистрировать углы изгиба не меньше чем в двух точках на прямоугольной консоли кантилевера и угол кручения в какой-то из них. Доказано аналитически, что одной оптимальной точкой является пересечение оси зонда с плоскостью консоли. Разработана методика расчета оптимального положения другой точки. Проведен эксперимент по картированию силы и вектора смещения, получено удовлетворительное согласие с теорией.

Ключевые слова: атомно-силовая микроскопия, прямые измерения пространственных компонент силы, кантилевер, оптический рычаг.

DOI: 10.21883/JTF.2021.06.50877.303-20

\section{Введение}

В атомно-силовой микроскопии (АСМ) $[1,2]$ поверхность образца исследуется твердотельным зондом с радиусом закругления кончика несколько нанометров. Зонд расположен на свободном конце упругой консоли субмиллиметровых размеров (вся конструкция называется кантилевером), деформации которой измеряются для контроля силы взаимодействия зонд-образец [2]. В таких востребованных применениях АCM, как метрология боковых стенок микроструктур [3], манипуляция микро- и наночастицами $[4,5]$, силовая микроскопия пьезоотклика [6], важно знать величину и направление перемещения кончика зонда и силы взаимодействия зонд-образец. Для этого нужно детектировать три независимых параметра, характеризующих деформацию консоли $[2,7,8]$. Это позволяет пока редкая схема регистрации деформаций консоли оптического рычага (ОР) + интерферометра (И) $[7,9,10]$, комбинирующая методы ОР [11] и И [12]. В выбранной на консоли точке первым методом детектируют углы изгиба и кручения, а вторым - величину изгиба. Также все пространственные компоненты силы взаимодействия и вектора смещения можно определить только методом OP $[8,13]$, если кроме углов изгиба и кручения в одной точке на консоли измерить угол изгиба в какой-то другой точке.

Разработчики схемы ОР + И $[9,10]$ фокусировали и лазер ОР, и лазер И на свободном конце консоли. Оптимальность такого положения фокуса при определении вектора силы взаимодействия зонд-образец не рас- сматривалась. В экспериментальном исследовании [8], использовавшем только метод ОР, докладывалось, что свободный конец и примерно середина консоли являются оптимальными положениями фокуса. Авторы [13], учтя в расчетах только разброс значений угла изгиба консоли, оценили графически, что минимальная ошибка в измерениях компонент силы достигается при выборе фокуса в нормированных на длину консоли положениях: около 0.46 и 1.00 .

В АСМ метод ОР позволяет измерять угол отклонения консоли в заданной точке с точностью $10^{-7}-10^{-6} \mathrm{rad}[2]$. Для консоли типичной длины $200 \mu \mathrm{m}$ ошибка измерений величины изгиба в той же точке будет менее одного ангстрема. Положение фокуса лазера схемы ОР обычно измеряется с точностью единицы, десятки микрометров $[14,15]$. Неопределенность положения фокуса, оказываясь на 5-6 порядков больше разброса величины изгиба консоли, может доминировать в ошибке измерений компонент силы. Это снижает значимость результата работы [13], рассмотревшей случай, когда эту ошибку определяет только разброс значений угла изгиба.

В настоящей работе для атомно-силового микроскопа со схемой ОР был аналитически рассмотрен общий случай оптимизации измерений компонент силы и тензорно связанного $[15,16]$ с ней вектора смещения „идеального кантилевера“ (зонд такого кантилевера не деформируется [15]). Доказано, что минимум ошибки измерений достигается в двух особенных точках фокуса лазера. Положение первой зависит от следующих величин: отношение ошибок измерений угла изгиба (про- 
изводной угла изгиба по вертикальному направлению) прямоугольной консоли и координаты фокуса лазера $\mathrm{OP}$, пространственные компоненты силы или вектора смещения в плоскости изгиба (производные этих компонент по вертикальному направлению), высота зонда, длина консоли и ее изгибная жесткость. Вторая точка находится на свободном конце прямоугольной консоли, где крепится зонд. Используя полученные аналитические результаты, проведено экспериментальное картирование вектора смещения острия АСМ-зонда и действующей на него силы.

\section{1. Оптимальные положения фокуса лазера ОР на консоли для картирования силы взаимодействия зонд-образец
и вектора смещения
„идеального кантилевера“}

Пусть сила F приложена к кончику зонда АСМкантилевера (рис. 1). Две пространственные компоненты $F_{Y}$ и $F_{Z}$ вызывают изгиб консоли кантилевера на угол $\alpha$, а не указанная, перпендикулярная им третья компонента $F_{X}$ закручивает ее на угол $\beta$.

Если нормированная координата фокуса лазера на консоли $x=Y / l_{C}$, высота зонда $l_{T}$, длина консоли $l_{C}$, a ее изгибная жесткость $k_{C}$, то $\beta(x)=$ $=-\left(2 l_{T} F_{X} / k_{C} l_{C}^{2}\right) x \quad[15,16] . \quad$ Введем разброс в измерениях $x$ и $\beta$, дисперсии $\sigma_{x}^{2}$ и $\sigma_{\beta}^{2}$. Дисперсия $\sigma_{F_{X}}^{2}$ убывает на отрезке $x \in[0 ; 1]$ и минимальна в $x=1$, где $\sigma_{F_{X}}=k_{C} l_{C}^{2} /\left(2 l_{T}\right) \sqrt{\beta^{2}(1) \sigma_{x}^{2}+\sigma_{\beta}^{2}}$. С другой стороны, с $\beta$ можно связать компоненту $X^{C}$ вектора смещения $\mathbf{r}^{C}$,идеального кантилевера“ $[15]: \beta(x)=$ $=-\left[2 l_{T} X^{C} /\left(\left(2 \lambda^{2}+\delta^{2}\right) l_{C}^{2}\right)\right] x$, где введены $\lambda=l_{T} / l_{C}$ и отношение толщины $t$ к ширине $w$ консоли $\delta=t / w$. Как и для $F_{X}$, дисперсия $X^{C}$ минимальна тоже в $x=1$, где $\sigma_{X^{c}}=\left(2 \lambda^{2}+\delta^{2}\right) l_{C}^{2} /\left(2 l_{T}\right) \sqrt{\beta^{2}(1) \sigma_{x}^{2}+\sigma_{\beta}^{2}}$.

Угол изгиба консоли $\alpha$ - это квадратичная функция $x$, коэффициенты которой выражаются через $F_{Y}$ и $F_{Z}$ либо через компоненты $Y^{C}$ и $Z^{C}$ вектора $\mathbf{r}^{C}$ (рис. 1), [15]:

$$
\begin{gathered}
\alpha(x)=\frac{3 F_{Z}+3 \lambda F_{Y}}{k_{C} l_{C}} x-\frac{3 F_{Z}}{2 k_{C} l_{C}} x^{2}=\left(\frac{6 Z^{C}}{l_{C}}-\frac{2 Y^{C}}{l_{T}}\right) x \\
-\left(\frac{6 Z^{C}}{l_{C}}-\frac{3 Y^{C}}{l_{T}}\right) x^{2} .
\end{gathered}
$$

Обозначив $\alpha(x)=y$, запишем (1) в общем виде

$$
\begin{gathered}
y=a x-b x^{2} ; a=\bar{a}=\frac{3 F_{Z}+3 \lambda F_{Y}}{k_{C} l_{C}}=\underline{a}=\frac{6 \lambda Z^{C}-2 Y^{C}}{l_{T}}, \\
b=\bar{b}=\frac{3 F_{Z}}{2 k_{C} l_{C}}=\underline{b}=\frac{6 \lambda Z^{C}-3 Y^{C}}{l_{T}} .
\end{gathered}
$$

Буквенные величины, относящиеся к смещению, условно, подчеркнуты, а к силе - приведены с чертой

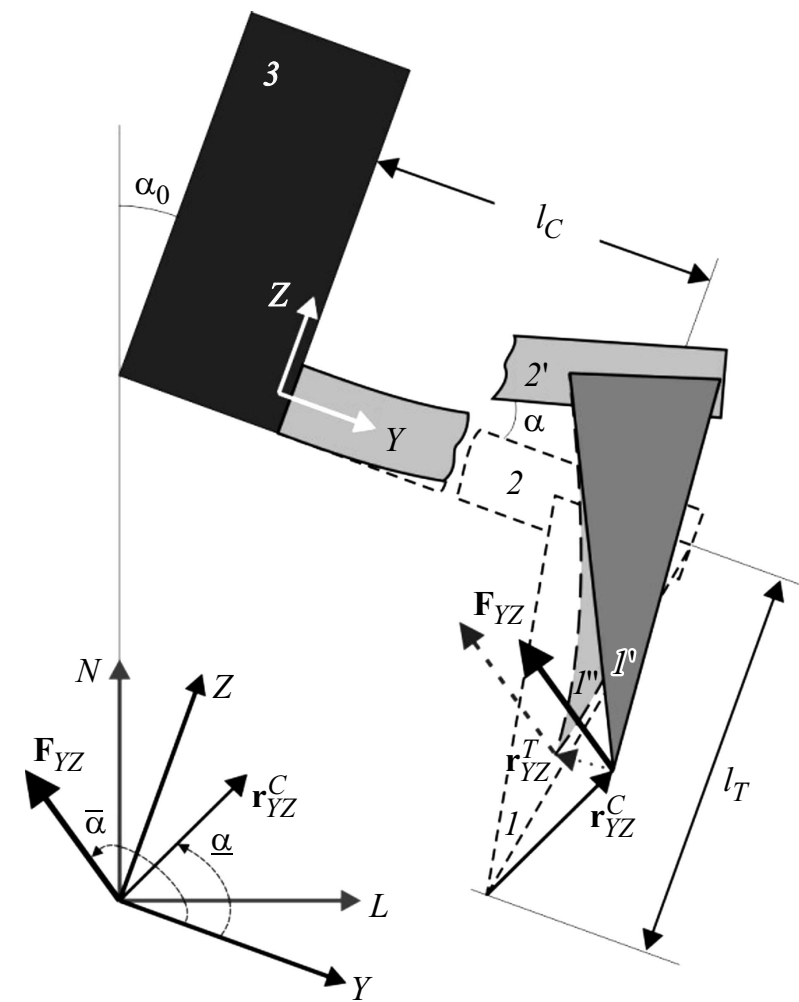

Рис. 1. Деформации АСМ-кантилевера. В отсутствие силы зонд (1) и консоль (2) не изогнуты. После приложения силы $\mathbf{F}_{Y Z}$ : зонд $\left(1^{\prime \prime}\right)$ кантилевера деформировался, $\mathbf{r}_{Y Z}^{T}$, консоль $\left(2^{\prime}\right)$ деформировалась, условно недеформируемый зонд $\left(1^{\prime}\right)$ „идеального кантилевера“ сместился, $\mathbf{r}_{Y Z}^{C}$; недеформируемый держатель кантилевера (3) остался на месте. Системы координат $X Y Z$ и $X L N$ привязаны к кантилеверу и соответственно к сканеру (ось $X$ направлена на читателя); $\alpha_{0}$ - угол отклонения держателя кантилевера от вертикали (например, $\approx 20^{\circ}$ в приборах НТ-МДТ СИ, Россия и $\approx 10^{\circ}-$ Bruker, США); $\alpha$ - угол изгиба консоли в выбранной точке; $\bar{\alpha}$ и $\underline{\alpha}-$ полярные углы векторов $\mathbf{F}_{Y Z}$ и $\mathbf{r}_{Y Z}^{C} ; l_{C}-$ длина консоли; $l_{T}$ - высота зонда.

сверху. Ниже другие величины, обозначенные одинаковой буквой, но с чертой сверху или снизу, могут отличаться друг от друга.

Для однозначного определения коэффициентов $a$ и $b$ в (2) необходимо измерить значения $y_{1}$ и $y_{2}$ в двух разных положениях на консоли, $x_{1}$ и $x_{2}$ :

$$
\left\{\begin{array}{l}
a=\frac{1}{\left(x_{2}-x_{1}\right)}\left(x_{2} \frac{y_{1}}{x_{1}}-x_{1} \frac{y_{2}}{x_{2}}\right), \\
b=\frac{1}{\left(x_{2}-x_{1}\right)}\left(\frac{y_{1}}{x_{1}}-\frac{y_{2}}{x_{2}}\right) .
\end{array}\right.
$$

Сформулируем математическую задачу: для заданных ошибок измерений $\sigma_{y_{1}}, \sigma_{y_{2}}, \sigma_{x_{1}}, \sigma_{x_{2}}$ найти такие $x_{1(\text { extr })}$ и $x_{2(\text { extr })}$, чтобы $\sigma_{a}$ и $\sigma_{b}$, ошибки определения $a$ и $b$ по формулам (3) были минимальными.

Частный случай этой задачи $\left(\sigma_{y_{1}}=\sigma_{y_{2}}, \sigma_{x_{1}}=\sigma_{x_{2}}\right)$ решен в разд. 3 Математическое приложение. Для общ- 
Таблица 1. Коэффициенты полиномов $\bar{p}=\sum_{i=0}^{4} \bar{a}_{i} v^{i}$ и $\underline{p}=\sum_{i=0}^{4} \underline{a}_{i} v^{i}$, корни которых на отрезке $v \in[0 ; 1]$ соответствуют $\bar{x}_{1(\text { extr })}(\bar{\alpha})$ и $\underline{x}_{1(\text { extr })}(\underline{\alpha})$

\begin{tabular}{l|l|l|l}
\hline $\bar{a}_{0}$ & $-(\sin \bar{\alpha}+\lambda \cos \bar{\alpha})^{2}-\bar{C}^{2}$ & $\bar{a}_{0}$ & $-(3 \lambda \sin \underline{\alpha}-\cos \underline{\alpha})^{2}-\underline{C}^{2}$ \\
\hline $\bar{a}_{1}$ & $(\sin \bar{\alpha}+\lambda \cos \bar{\alpha})(3 \sin \bar{\alpha}+2 \lambda \cos \bar{\alpha})+2 \bar{C}^{2}$ & $\underline{a}_{1}$ & $(3 \lambda \sin \underline{\alpha}-\cos \underline{\alpha})(12 \lambda \sin \underline{\alpha}-5 \cos \underline{\alpha})+2 \underline{C}^{2}$ \\
\hline $\bar{a}_{2}$ & $-3 \sin \bar{\alpha}(\sin \bar{\alpha}+\lambda \cos \bar{\alpha})$ & $\underline{a}_{2}$ & $-9(2 \lambda \sin \underline{\alpha}-\cos \underline{\alpha})(3 \lambda \sin \underline{\alpha}-\cos \underline{\alpha})$ \\
\hline $\bar{a}_{3}$ & $0.81\left(\bar{C}^{2}+\lambda^{2} \cos ^{2} \bar{\alpha}\right)+\sin ^{2} \bar{\alpha}$ & $\underline{a}_{3}$ & $0.34\left(\underline{C}^{2}+(3 \lambda \sin \underline{\alpha}-2 \cos \underline{\alpha})^{2}\right)+9(2 \lambda \sin \underline{\alpha}-\cos \underline{\alpha})^{2}$ \\
\hline $\bar{a}_{4}$ & $0.19\left(\bar{C}^{2}+\lambda^{2} \cos ^{2} \underline{\alpha}\right)$ & $\underline{a}_{4}$ & $0.66\left(\bar{C}^{2}+(3 \lambda \sin \underline{\alpha}-2 \cos \underline{\alpha})^{2}\right)$
\end{tabular}

Приме чан ие. Измеряется угол изгиба консоли $\alpha=y, \bar{C}=\left(k \times k_{C} l_{C} /\left(3\left|\mathbf{F}_{Y Z}\right|\right)\right.$ и $\underline{C}=k \times l_{C} /\left(2\left|\mathbf{r}_{Y Z}^{C}\right|\right)$; дифференциальный угол изгиба консоли $\delta \alpha / \delta N=y, \bar{C}=k \times k_{C} l_{C} /\left(3\left|\delta \mathbf{F}_{Y Z} / d N\right|\right)$ и $\underline{C}=k \times l_{C} /\left(2 \mid\left(\delta \mathbf{r}_{Y Z}^{C} / \delta N \mid\right)\right.$. Углы $\bar{\alpha}$ и $\underline{\alpha}$ определяют в плоскости $Y Z$ направления силы взаимодействия зонд-образец и смещения зонда „идеального кантилевера“.

ности анализировалась линейная комбинация дисперсий $M\left(x_{1}, x_{2}\right)=\cos ^{2} \theta \sigma_{a}^{2}+\sin ^{2} \theta \sigma_{b}^{2}$ (см. (П5)); расчет вспомогательного параметра $\theta$ показан в разд. 3.5. Доказано, что одна координата минимума $M\left(x_{1}, x_{2}\right) x_{2(e x t r)}=1$, a

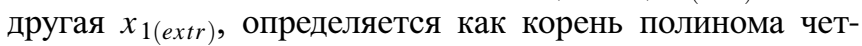
вертой степени (см. (П41), (П16) и (П26), (П47а)):

$$
\begin{aligned}
P & =\sum_{i=0}^{4} a_{i} v^{i}=\cos ^{2} \theta\left(k^{2}+(2 b-a)^{2}\right) v^{4} \\
& +\left(\sin ^{2} \theta\left(k^{2}+(2 b-a)^{2}\right)+4 b^{2}\right) v^{3}-6 a b v^{2} \\
& +2\left(k^{2}+a b+a^{2}\right) v-\left(k^{2}+a^{2}\right)=0, v \in[0 ; 1],
\end{aligned}
$$

где коэффициент $k=\sigma_{y} / \sigma_{x}, \sigma_{y}=\sigma_{\alpha}$ и $\sigma_{x}$ - это ошибки измерений угла изгиба и нормированной координаты фокуса. Корень (4a) сильно зависит от $a / k$ и $b / k$, а от $\theta$ слабо (см. разд. 3 ).

Коэффициенты $\bar{a}$ и $\bar{b}$ в (2) выражаются через амплитуду вектора $\left|\mathbf{F}_{Y Z}\right|$ и угол $\bar{\alpha}$ между ним и направлением $+Y$ (рис. 1):

$$
\left\{\begin{array}{l}
\bar{a}=\frac{3\left|\mathbf{F}_{Y Z}\right|}{k_{C} l_{C}}(\sin \bar{\alpha}+\lambda \cos \bar{\alpha}), \\
\bar{b}=\frac{3\left|\mathbf{F}_{Y Z}\right|}{2 k_{C} l_{C}} \sin \bar{\alpha} .
\end{array}\right.
$$

Подстановка (4b) и значения $\bar{\theta}$ из (П48) в (4a) дает полином $\bar{p}=\sum_{i=0}^{4} \bar{a}_{i} v^{i}$, формулы коэффициентов $\bar{a}_{i}$ которого внесены в левую часть табл. 1. У этого полинома на отрезке [0;1] всегда есть корень $v=\bar{x}_{1(\text { extr) }}$, и он является функцией $\bar{\alpha}$.

Угловые диаграммы $\bar{x}_{1(e x t r)}(\bar{\alpha})$ приведены на рис. $2, a$. Их форма зависит от величины параметра $\bar{C} \propto k$ (табл. 1). Когда $\bar{C}=k=0$, диаграмма напоминает заостренную сверху и снизу восьмерку. Случай соответствует $\sigma_{y} / \sigma_{x}=\sigma_{\alpha} / \sigma_{x}=0$, когда ошибка определения значений компонент силы зависит только от разброса положения фокуса лазера ОР. Для $1 / \bar{C}=1 / k=0, \sigma_{x} / \sigma_{y}=0$, только разброс значений угла изгиба определяет эту ошибку [13], и диаграмма - это окружность $x \approx 0.464$.
Если на рис. 2, $a$ через начало координат провести прямую вдоль вектора $\mathbf{F}_{Y Z}$, то в точке на расстоянии $\bar{x}_{1(e x t r)}$ от центра она пересечет диаграмму с характеризующим эксперимент значением $\bar{C}$. Вместе c $x_{2(e x t r)}=1-$ это два оптимальных нормированных положения фокуса лазера ОР для определения $F_{Z}$ и $F_{Y}$ компонент, а также (см. начало этого раздела) $F_{X}$ компоненты силы.

Если направление силы неизвестно, можно использовать точку пересечения диаграммы $\bar{x}_{1(\text { extr })}(\bar{\alpha})$ с прямой, идущей через начало координат вдоль локальной нормали к поверхности образца, и измерить $F_{Y}$ и $F_{Z}$ приблизительно. Определенный таким образом приблизительный вектор $\mathbf{F}_{Y Z}$ дает следующее приближение для направления секущей прямой, и производится уточняющее измерение $F_{Y}$ и $F_{Z}$.

Если вектор $\mathbf{r}_{Y Z}^{C}$ задать амплитудой $\left|\mathbf{r}_{Y Z}^{C}\right|$ и углом $\underline{\alpha} \mathrm{c}$ направлением $+Y$ (рис. 1), выражения для $\underline{a}$ и $\underline{b}$ в (2) преобразуются к

$$
\left\{\begin{array}{l}
\underline{a}=\frac{2\left|\mathbf{r}_{Y Z}^{C}\right|}{\lambda l_{C}}(3 \lambda \sin \underline{\alpha}-\cos \underline{\alpha}), \\
\underline{b}=\frac{3\left|\mathbf{r}_{Y Z}^{C}\right|}{\lambda l_{C}}(2 \lambda \sin \underline{\alpha}-\cos \underline{\alpha}) .
\end{array}\right.
$$

Используя в (4a) $\underline{\theta}$ из (П49) и (4c), были получены (см. правую часть табл. 1) коэффициенты полинома $\underline{p}=\sum_{i=0}^{4} \underline{a}_{i} v^{i}$ - для расчетов корня $v=\underline{x}_{1(\text { extr })}(\underline{\alpha})$.

Диаграммы $\bar{x}_{1(\text { extr) }}(\bar{\alpha})$ и $\underline{x}_{1(\text { extr })}(\underline{\alpha})$ с конечными значениями параметров $\bar{C}$ и $\underline{C}$ (рис. 2) отличаются. В частности, для почти всех направлений силы $\underline{x}_{1(\text { extr })}>0.464$, а для большинства направлений смещения $\underline{x}_{1(\text { extr }}<0.464$.

Определим связь между $\bar{\alpha}$ и $\underline{\alpha}$. В (4b) и (4c) $\bar{a}=\underline{a}$ и $\bar{b}=\underline{b}$, откуда получаем:

$$
\operatorname{tg} \underline{\alpha}=(3 \lambda+2 \operatorname{tg} \bar{\alpha}) /\left(6 \lambda^{2}+3 \lambda \operatorname{tg} \bar{\alpha}\right) .
$$

Например, для $\lambda=0.2$, отрезки $\underline{\alpha} \in[\operatorname{arctg}(1 / 2 \lambda)$; $\operatorname{arctg}(2 / 3 \lambda)] \cong\left[68.2^{\circ} ; 73.3^{\circ}\right]$ и $\bar{\alpha}\left[0^{\circ} ; 90^{\circ}\right]$, соответствуя 

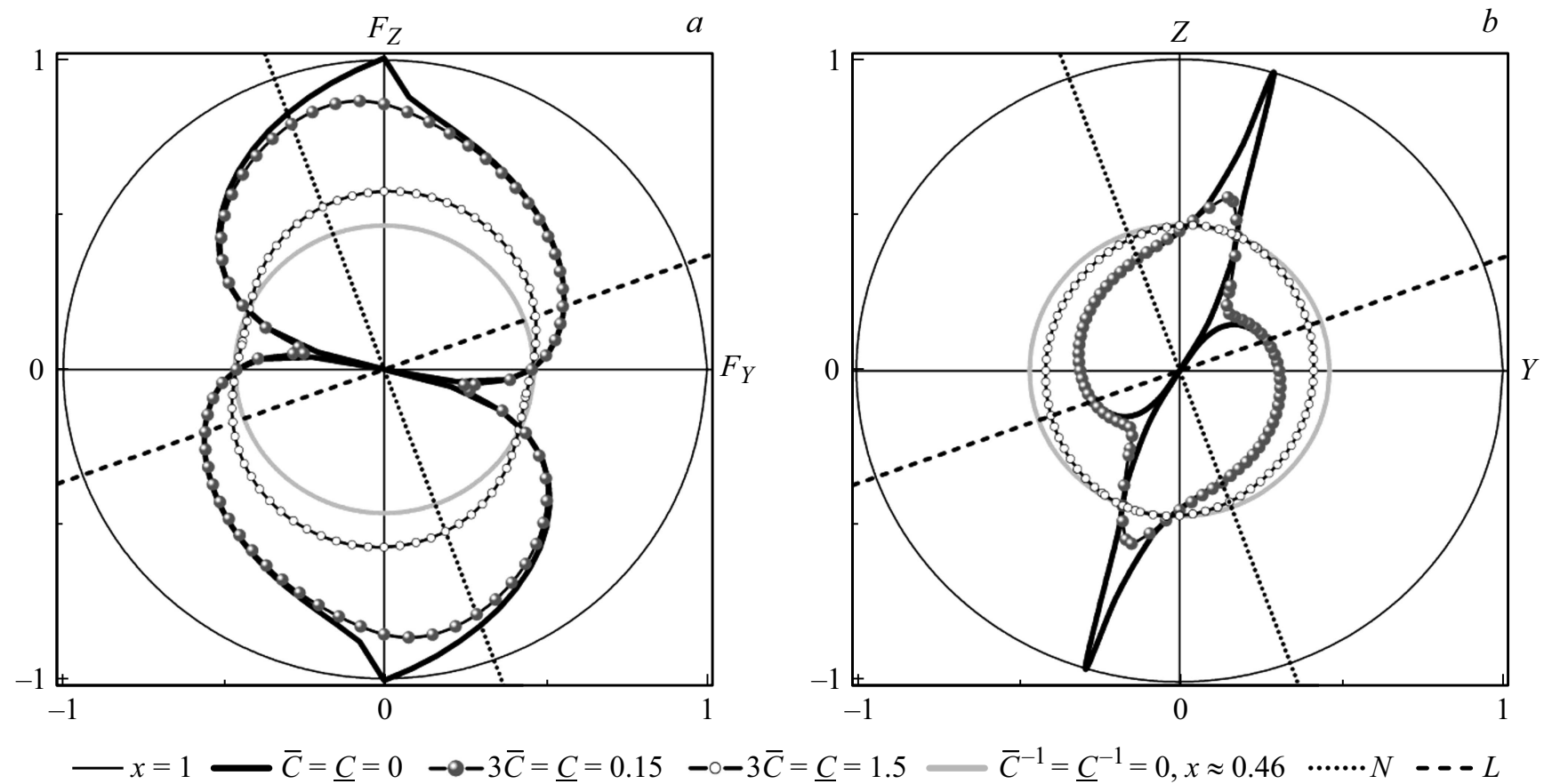

Рис. 2. $a-$ диаграммы $\bar{x}_{1(\text { extr })}(\bar{\alpha})$ для четырех значений параметра $\bar{C}$ (табл. 1 и формулы $\left.(7)\right) ; \bar{\alpha}-$ полярный угол $\mathbf{F}_{Y Z .} b-$ диаграммы $\underline{x}_{1(\text { extr })}(\underline{\alpha})$ для 4-х значений параметра $\underline{C} ; \underline{\alpha}-$ полярный угол $\mathbf{r}_{Y Z}^{C}$. Диаграмма $x_{2(\text { extr })}-$ окружность $x=1$; вертикаль, горизонталь - прямые $N, L$. Расчеты для кантилевера с $\lambda=0.2$ и угла $\alpha_{0}=20^{\circ}$ велись в Mathcad 15 (PTC, США).

друг другу, отличаются длиной и положением начальной точки. Такого рода деформации и смещения при отображении $\bar{\alpha}$ на $\underline{\alpha}$ проявляются в отличиях диаграмм $\bar{x}_{1(\text { extr })}(\bar{\alpha})$ для $\mathbf{F}$ и $\underline{x}_{1(\text { extr })}(\underline{\alpha})$ для $\mathbf{r}^{C}$ (рис. 2$)$.

Диаграммы $\bar{x}_{1(\text { extr) }}(\bar{\alpha})$ применимы для оптимизации измерений компонент силы с помощью реального кантилевера с прямоугольной консолью. Диаграммы $\underline{x}_{1(\text { extr })}(\underline{\alpha})$ предназначены для „идеального кантилевера“. У жестких кантилеверов при работе в контактном режиме смещение острия зонда заметно отличается от смещения зонда „идеального кантилевера“ $\mathbf{r}_{Y Z}^{C}$, так как величина изгибной жесткости кремниевого зонда $(\sim 10 \mathrm{~N} / \mathrm{m}[15,17,18])$ сопоставима с жесткостью консоли. На схеме реального кантилевера на рис. 1 это демонстрирует вектор деформации зонда $\mathbf{r}_{Y Z}^{T}$.

\section{2. Экспериментальная верификация результатов}

\section{1. АСМ измерения $\alpha(N), \beta(N), \delta \alpha(x) / \delta N$, $\delta \beta(x) / \delta N$ зависимостей}

В схеме OP сигнал DFL (deflection), разности фототоков в верхней и нижней половинах фотодетектора (ФД) линейно связан с углом изгиба консоли $\alpha$, а сигнал LF (lateral force), разности фототоков в левой и правой половинах ФД - с углом кручения консоли $\beta$ [19]. Сигналы DFL и LF выводятся в условных единицах. Для отображения в радианах нужно определить коэффициенты перевода $C_{1}=\delta \alpha / \delta \mathrm{DFL}$ и $C_{1^{*}}=\delta \beta / \delta \mathrm{LF}$.
В АCM можно детектировать зависимости $\operatorname{DFL}(N)$ и $\operatorname{LF}(N)$ (вертикальное смещение образца $N$ регулируется сканером). Обычно это линейные зависимости с коэффициентами наклона $C_{2}=\delta \mathrm{DFL} / \delta N$ и $C_{2 *}=\delta \mathrm{LF} / \delta N$.

Произведения $C_{1} C_{2}$ и $C_{1 *} C_{2 *}$ составляют дифференциальные сигналы $\delta \alpha / \delta N$ и $\delta \beta / \delta N$, которые, как и сигналы DFL и LF, зависят от $x=Y / l_{C}$, нормированной координаты фокуса лазера. При анализе данных $\delta \alpha(x) / \delta N$ и $\delta \beta(x) / \delta N$ вектора и компоненты силы и смещения в соотношениях теоретического раздела следует заменить на их производные по $N$. Например, силу $\mathbf{F}_{Y Z}$ и смещение $\mathbf{r}_{Y Z}^{C}$ замещают дифференциальные сила $\delta \mathbf{F}_{Y Z} / \delta N$ и смещение $\delta \mathbf{r}_{Y Z}^{C} / \delta N$.

Использовался атомно-силовой микроскоп Интегра Аура (НТ-МДТ СИ) в конфигурации сканирование образцом. Положение ФД в горизонтальной плоскости регулировали подпружиненные винты с шагом резьбы $0.35 \mathrm{~mm}$. В результате изгиба консоли изменения угла отражения света лазера ОР от нее в два раза больше, чем сам угол изгиба [2]. Угол между лучами из вершины на кантилевере в концы отрезка, отмечающего два положения центра ФД, обратно пропорционален длине оптического рычага, $25 \mathrm{~mm}$ [15]. С учетом наклона плоскости ФД на оборот винта, двигающего его вдоль консоли, приходится угол $\delta \alpha=\cos 20^{\circ} \cdot 0.35 /(2 \cdot 25) \cong 6.58 \cdot 10^{-3} \mathrm{rad}$, а на оборот винта, двигающего его поперек консоли, - угол $\delta \beta=0.35 / 50=7 \cdot 10^{-3} \mathrm{rad}$. Используя это, $C_{1}$ и $C_{1 *}$ определялись с относительной точностью около $4 \%$ (1/24 оборота винта). 
Положение фокуса лазера на консоли, $Y$, измерялось по углу поворота винта, двигающего столик с держателем кантилевера вдоль консоли. С учетом плеча силы $(\approx 51 \mathrm{~mm})$, которой винт толкает столик, и расстояния от оси вращения столика до кантилевера $(\approx 34 \mathrm{~mm})$, один оборот винта с шагом резьбы $0.35 \mathrm{~mm}$ смещает кантилевер на $\approx 240 \mu \mathrm{m}$. Это позволяло измерять $Y$ с точностью $10 \mu \mathrm{m}(1 / 24$ оборота винта). Линия закрепления консоли на чипе, $Y=0$, определялась по падению в два раза суммарной интенсивности излучения лазера, отраженного консолью в четыре секции ФД (сигнал Laser) $[14,15,18]$. Длина $l_{C}$ измерялась с точностью $1 \mu \mathrm{m}$ в оптическом микроскопе по расстоянию от линии закрепления до пересечения оси зонда и консоли.

В теоретическом разделе отмечалось, что угол $\beta$ (либо дифференциальный угол $\delta \beta / \delta N$ ) однозначно задает $F_{X}\left(\delta F_{X} / \delta N\right)$ и $X^{C}\left(\delta X^{C} / \delta N\right)$. Ниже рассматриваются особенности более сложных измерений и трактовки значений $\delta \alpha / \delta N$.

\section{2. Расчет $\bar{x}_{1(\text { extr })}$ и $\underline{x}_{1(\text { extr) }}$, характеризующие эксперимент параметры $\bar{C}$ и $\underline{C}$}

Расчет $\bar{x}_{1(\text { extr })}$ и $\underline{x}_{1(\text { extr })}$ как корней полиномов табл. 1 можно проводить, если выполнены два условия: 1) $\left.\sigma_{x_{1}}=\sigma_{x_{2}}=\sigma_{x}, 2\right) \sigma_{y_{1}}=\sigma_{y_{2}}=\sigma_{y}$, - случай равных дисперсий, см. (П3). В эксперименте дисперсии измеряемых величин $x=Y / l_{C}, y(x)=\delta \alpha(x) / \delta N=C_{1} C_{2}(x)$ и отношение $k$, определяющее $\bar{C}$ и $\underline{C}$, рассчитывались следующим образом:

$$
\left\{\begin{array}{l}
\sigma_{x}=\frac{Y}{l_{C}} \sqrt{\frac{\sigma_{Y}^{2}}{Y^{2}}+\frac{\sigma_{l_{C}}^{2}}{l_{C}^{2}}} \approx \frac{\sigma_{Y}}{l_{C}}=\mathrm{const} \\
\sigma_{y}=\frac{\delta \alpha}{\delta N} \sqrt{\frac{\sigma_{C_{1}}^{2}}{C_{1}^{2}}+\frac{\sigma_{C_{2}}^{2}}{C_{2}^{2}}} \approx C_{2} \sigma_{C_{1}}=\frac{\delta \alpha}{\delta N} \frac{\sigma_{C_{1}}}{C_{1}} \\
k=\frac{\sigma_{y}}{\sigma_{x}} \approx \frac{\delta \alpha}{\delta N} \frac{l_{C} \sigma_{C_{1}}}{C_{1} \sigma_{Y}}
\end{array}\right.
$$

Первое условие выполнялось строго, так как $\sigma_{Y} \cong 10 \mu \mathrm{m}$ и от $x$ не зависит, $\sigma_{l_{C}}<1 \mu \mathrm{m}, Y \leq l_{C}$. Поскольку $C_{1}$ не зависит от $x$, второе условие выполняется для $\sigma_{C_{2}}=$ const, когда $\sigma_{C_{1}} / C_{1} \ll \sigma_{C_{2}} / C_{2}$. Однако из-за фиксированного $\sigma_{C_{1}} / C_{1}=1 / 24$, это приводит к большой $\sigma_{y}$. Добиваясь $\sigma_{C_{2}} / C_{2} \ll \sigma_{C_{1}} / C_{1}$, увеличив число измерений, мы снижали $\sigma_{y}$ до предельного значения, ограниченного отношением сигнал/шум $(\delta \alpha / \delta N) / \delta_{y}=$ $=C_{1} / \sigma_{C_{1}}=24$. В результате из-за отличий $\delta \alpha\left(x_{1}\right) / \delta N$ и $\delta \alpha\left(x_{2}\right) / \delta N$ второе условие строго не выполнялось.

Рассмотрим экспериментальную зависимость $\alpha(N)$ на вставке рис. 3,a. Анализ сигнала на линейном, контактном участке дает: $\sigma_{\alpha} \approx 2.8 \mu \mathrm{rad}$; шумкоординаты $\sigma_{N} \approx 0.1 \mathrm{~nm}$; высота и ширина участка $150 \mu \mathrm{rad}$ и $18 \mathrm{~nm}$. Таким образом, $\sigma_{C_{2}} / C_{2}=$ $=\sqrt{(2.8 / 150)^{2}+(1 / 180)^{2}} \ll \sigma_{C_{1}} / C_{1}=1 / 24$. Следова-
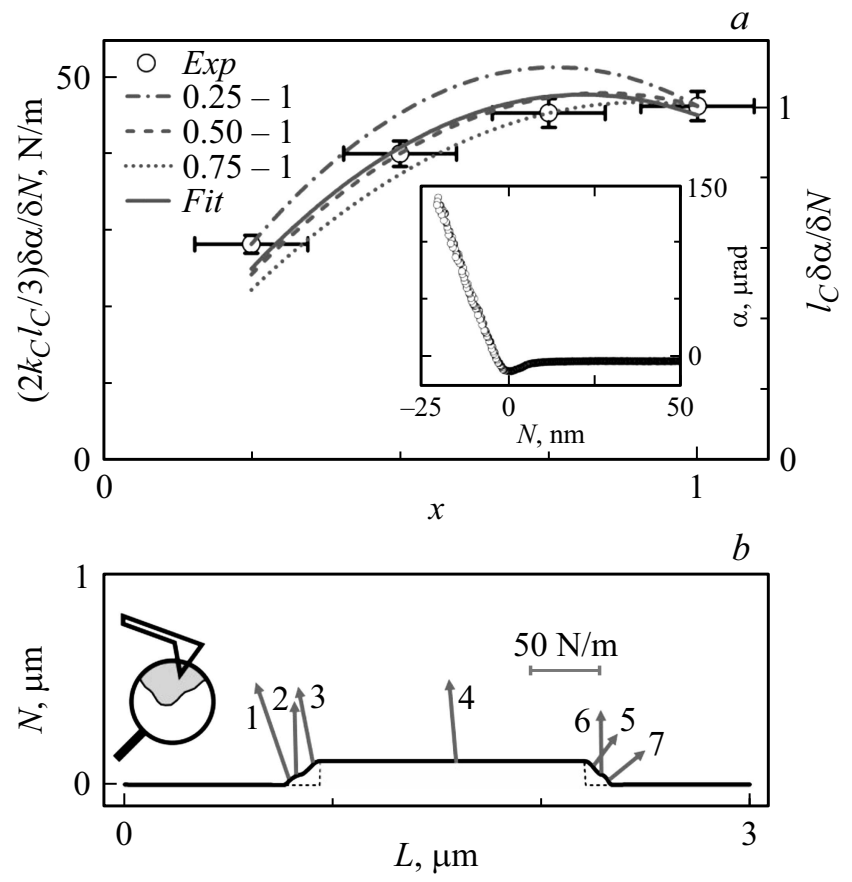

Рис. 3. $a-$ профиль дифференциального угла изгиба консоли для контакта АCM-зонда на горизонтальном участке выступа решетки TGZ2, Exp. Модельные зависимости, точно проходящие через две точки измерений: $x_{1}=0.25$ и $x_{2}=1,0.25-1$; $x_{1}=0.5$ и $x_{2}=1,0.5-1 ; x_{1}=0.75$ и $x_{2}=1,0.75-1$. Подгонка модельной зависимости ко всему профилю, Fit. Вставка: разгрузочная кривая $\alpha(N), x=0.5 . b-$ измеренный профиль высоты выступа (отображается полусумма сигналов в прямом и обратном направлениях сканирования), - сплошная линия; истинный рельеф - пунктир. Схема слева: расположение кантилевера, возможная форма острия. Стрелки 1-7 на участках разного наклона изображают вектор $\delta \mathbf{F}_{Y Z} / \delta N$, табл. 2 , $x_{1}=0.75$ и $x_{2}=1$. Углы между направлением $+L$ и стрелками меньше $\bar{\alpha}$ табл. 2 на $\alpha_{0}$. Использовался кантилевер RTESPA$300\left(l_{C}=105 \mu \mathrm{m}, \lambda=0.2, k_{C}=69 \mathrm{~N} / \mathrm{m}\right) ; \alpha_{0}=20^{\circ}$.

тельно, можно не учитывать вклад $\sigma_{C_{2}} / C_{2}$ в $\sigma_{y}$ и $\sigma_{y} \approx \delta \alpha / \delta N\left(\sigma_{C_{1}} / C_{1}\right)$, как приведено в $(6)$.

Используя выражение для $k$ в (6), получаются необходимые при расчете коэффициентов полиномов в табл. 1 формулы для $\bar{C}$ и $\underline{C}$ :

$$
\left\{\begin{array}{l}
\bar{C} \cong \frac{\sigma_{C_{1}} / C_{1}}{\sigma_{Y} / l_{C}} \frac{k_{C} l_{C}(\delta \alpha / \delta N)}{3\left|\delta \mathbf{F}_{Y Z} / \delta N\right|} \\
\underline{C} \cong \frac{\sigma_{C_{1}} / C_{1}}{\sigma_{Y} / l_{C}} \frac{\lambda l_{C}(\delta \alpha / \delta N)}{2\left|\delta \mathbf{r}_{Y Z}^{C} / \delta N\right|}
\end{array}\right.
$$

В эксперименте $l_{C}=1.05 \cdot 10^{-4} \mathrm{~m}$ и $\lambda=l_{T} / l_{C}=0.2$, согласно данным оптического микроскопа с объективом с $N A=0.8$; жесткость $k_{C}=69 \mathrm{~N} / \mathrm{m}$, определялась методом Садера [20]. Подставляя эти значения и $\sigma_{Y}=10^{-5} \mathrm{~m}, \sigma_{C_{1}} / C_{1}=1 / 24,\left|\delta \mathbf{F}_{Y Z} / \delta N\right| \approx k_{C}$, $l_{C}(\delta \alpha / \delta N) \approx 3 / 2,\left|\delta \mathbf{r}_{Y Z}^{C} / \delta N\right| \approx 1$ в (7), получаем характеризующие эксперимент оценочные значения параметров: $\bar{C} \approx 0.219$ и $\underline{C} \approx 0.066$. В качестве примера для 
вертикальной силы $\mathbf{F}_{Y Z}\left(\bar{\alpha}=110^{\circ}\right)$ были рассчитаны коэффициенты полинома в левой половине табл. 1 и определено, что $\bar{x}_{1(\text { extr })} \cong 0.67$. Согласно уравнению (5), направление вектора смещения, соответствующего вертикальной силе $\mathbf{F}_{Y Z}$, задается углом $\underline{\alpha} \approx 74^{\circ}$. Вычисляя коэффициенты полинома в правой половине табл. 1, было получено, что $\underline{x}_{1(\text { extr })} \cong 0.69$.

\section{3. Расчет компонент $\mathrm{F}$ и $\mathrm{r}^{C}$ по $\mathrm{ACM}$ данным}

Взяв в (2), вместо компонент силы и вектора смещения „идеального кантилевера“, дифференциальные компоненты, комбинируя результат с (3), можно связать их со значениями $y=\delta \alpha / \delta N$ в паре точек $x_{1}, x_{2}$ :

$$
\left\{\begin{array}{l}
\frac{\delta F_{Y}}{\delta N}=\frac{k_{C} l_{C}}{3 \lambda\left(x_{2}-x_{1}\right)}\left\{\left(x_{2}-2\right) \frac{y_{1}}{x_{1}}-\left(x_{1}-2\right) \frac{y_{2}}{x_{2}}\right\}, \\
\frac{\delta F_{Z}}{\delta N}=\frac{2 k_{C} l_{C}}{3\left(x_{2}-x_{1}\right)}\left\{\frac{y_{1}}{x_{1}}-\frac{y_{2}}{x_{2}}\right\}, \\
\frac{\delta Y^{C}}{\delta N}=\frac{\lambda l_{C}}{x_{2}-x_{1}}\left\{\left(x_{2}-1\right) \frac{y_{1}}{x_{1}}-\left(x_{1}-1\right) \frac{y_{2}}{x_{2}}\right\}, \\
\frac{\delta Z^{C}}{\delta N}=\frac{l_{C}}{6\left(x_{2}-x_{1}\right)}\left\{\left(3 x_{2}-2\right) \frac{y_{1}}{x_{1}}-\left(3 x_{1}-2\right) \frac{y_{2}}{x_{2}}\right\} .
\end{array}\right.
$$

По (8) рассчитывались данные для пар точек в табл. 2 и 3. По ортогональным компонентам силы и смещения определялись значения углов

$$
\begin{gathered}
\underline{\alpha}=\operatorname{arctg}\left(\left(\delta Z^{C} / \delta N\right) /\left(\delta Y^{C} / \delta N\right)\right), \\
\bar{\alpha}=\operatorname{arctg}\left(\left(\delta F_{Z} / \delta N\right) /\left(\delta F_{Y} / \delta N\right)\right)
\end{gathered}
$$

и амплитуд

$$
\begin{aligned}
& \left|\delta \mathbf{r}_{Y Z}^{C} / \delta N\right|=\sqrt{\left(\delta Y^{C} / \delta N\right)^{2}+\left(\delta Z^{C} / \delta N\right)^{2}}, \\
& \left|\delta \mathbf{F}_{Y Z} / \delta N\right|=\sqrt{\left(\delta F_{Y} / \delta N\right)^{2}+\left(\delta F_{Z} / \delta N\right)^{2}},
\end{aligned}
$$

которые также нужны для вычислений $\bar{C}$ и $\underline{C}$ по формулам (7).

Для всех точек измеренного профиля $\delta \alpha(x) / \delta N$ (рис. 3,a) и данные секций Fit в табл. 2 и 3, значения $\left|\delta \mathbf{r}_{Y Z}^{C} / \delta N\right|,\left|\delta \mathbf{F}_{Y Z} / \delta N\right|, \bar{\alpha}, \underline{\alpha}$ определялись как параметры подгонки зависимостями, следующими из уравнений $(2),(4 b)$ и $(4 c)$ :

$$
\begin{gathered}
l_{C} \frac{\delta \alpha(x)}{\delta N}=\left|\frac{\delta \mathbf{r}_{Y Z}^{C}}{\delta N}\right|[(6 \sin \underline{\alpha}-(2 / \lambda) \cos \underline{\alpha}) x \\
\left.-(6 \sin \underline{\alpha}-(3 / \lambda) \cos \underline{\alpha}) x^{2}\right] \\
\frac{2 k_{C} l_{C}}{3} \frac{\delta \alpha(x)}{\delta N}=\left|\frac{\delta \mathbf{F}_{Y Z}}{\delta N}\right|\left[(2 \sin \bar{\alpha}+2 \lambda \cos \bar{\alpha}) x-\cos \bar{\alpha} x^{2}\right] .
\end{gathered}
$$

Как правило, в контактных АСМ измерениях угол изгиба кантилевера $\alpha$ линейно связан с изменениями вертикального положения образца, координаты $N$. Произведения определенных по (8)-(10) дифференциальных компонент силы и смещения на $\Delta N$, ширину линейной, контактной области на кривой $\alpha(N)$ дают значения самих компонент.

\section{4. Результаты эксперимента и обсуждение}

Одномерная тестовая решетка TGZ2 прямоугольных выступов высотой $110 \mathrm{~nm}$, чередующихся с периодом $3 \mu \mathrm{m}$, исследовалась в атомно-силовом микроскопе. Кантилевер устанавливался поперек выступов (вставка на рис. $3, b)$. Топография рельефа измерялась в режиме прерывистого контакта. Профиль высоты выступа на рис. $3, b$ получен с помощью кантилевера RTESPA300 , резонировавшего на частоте $368 \mathrm{kHz}$ с рабочей амплитудой вибраций $8 \mathrm{~nm}$ (амплитуда свободных вибраций $10 \mathrm{~nm}$ ), лазер ОР был сфокусирован в точку $x=0.5$.

На вставке рис. 3, a показана разгрузочная зависимость $\alpha(N)$, измеренная на горизонтальном участке 4 выступа (рис. 3,b). Угловой коэффициент ее линейного участка (статический контакт зонд-образец), $\delta \alpha / \delta N \cong 150 \mu \mathrm{rad} / 18 \mathrm{~nm} \cong 8300 \mathrm{rad} / \mathrm{m}$, умноженный на длину консоли, $1.05 \cdot 10^{-4} \mathrm{~m}$, дает ординату $\cong 0.87$ точки с $x=0.5$ на профиле дифференциального угла изгиба (рис. $3, a$ ), правая ось. Ордината этой же точки на левой оси $0.87 \cdot 2 \cdot 69 / 3 \cong 40 \mathrm{~N} / \mathrm{m}$. Таким путем по средним угловым коэффициентам разгрузочно-нагрузочных зависимостей $\alpha(N)$ в 4-х значениях $x$ были построены профили дифференциального угла изгиба на всех семи участках рельефа выступа, указанных на рис. $3, b$.

Для расчета амплитуд $\left|\delta \mathbf{r}_{Y Z}^{C} / \delta N\right|,\left|\delta \mathbf{F}_{Y Z} / \delta N\right|$ и углов $\bar{\alpha}$, $\underline{\alpha}$ эти профили и все 28 значений дифференциального угла изгиба обрабатывались по методике разд. 2.3. Результаты сведены в табл. 2 и 3.

На вставке рис. 3, $a$ ширина линейной, контактной области зависимости $\alpha(N) \Delta N \cong 18 \mathrm{~nm}$. Умножая дифференциальные амплитуды в табл. 2 и 3 на $\Delta N$, получаем для пары $x_{1}=0.5, x_{2}=1$ на горизонтальном участке образца значения амплитуд $\left|\mathbf{F}_{Y Z}\right|=86 \cdot 18=1.548 \mu \mathrm{N}$ и $\left|\mathbf{r}_{Y Z}^{C}\right|=0.77 \cdot 18=13.86 \mathrm{~nm}$.

Проанализируем данные $\delta \mathbf{r}_{Y Z}^{C} / \delta N$ в табл. 2. Из геометрических соображений проекция этого вектора на ось $N, \quad\left(\delta \mathbf{r}_{Y Z}^{C} / \delta N\right)_{N}=\left|\delta \mathbf{r}_{Y Z}^{C} / \delta N\right| \sin \left(\underline{\alpha}-\alpha_{0}\right)$, не может быть больше единицы. В табл. 2 серым цветом залиты ячейки 3-х пар с некорректными, с этой точки зрения, данными. (В табл. 3 для дифференциальной силы $\delta \mathbf{F}_{Y Z} / \delta N$ соответствующие пары исключены из результатов.) У оставшихся корректных 3-х пар $x_{2}=1$ совпадает с теоретическим $x_{2(\text { extr) }}$, вторым оптимальным положением фокуса, строго обоснованным в разд. 3 .

Для этих пар и для данных в секции Fit значения угла $\underline{\alpha}$ лежат в диапазоне $\left[71.9^{\circ} ; 77.7^{\circ}\right]$ вокруг пика диаграммы (рис. $2, b)$ (на пике $\underline{\alpha} \cong 73.3^{\circ}$, согласно (5), для 
Таблица 2. Компоненты $\delta \mathbf{r}_{Y Z}^{C} / \delta N$, определенные по данным дифференциального угла изгиба консоли $\delta \alpha / \delta N$ для шести пар ${ }^{1}$ нормированных позиций фокуса лазера системы ОР и по всем данным ${ }^{2} \delta \alpha(x) / \delta N$ измеренного профиля

\begin{tabular}{|c|c|c|c|c|c|c|c|c|}
\hline \multirow{2}{*}{$\begin{array}{l}x_{1} \\
x_{2}\end{array}$} & \multirow{2}{*}{ Компоненты } & \multicolumn{7}{|c|}{ Участки разного наклона на профиле высоты, рис. $3, b$} \\
\hline & & 1 & 2 & 3 & 4 & 5 & 6 & 7 \\
\hline 0.75 & $\left|\delta \mathbf{r}_{Y Z}^{C} / \delta N\right|$ & 0.67 & 0.68 & 0.61 & 0.73 & 0.45 & 0.59 & 0.46 \\
\hline 1 & $\underline{\alpha},{ }^{\circ}$ & 75.0 & 74.0 & 74.5 & 74.2 & 72.3 & 74.0 & 71.9 \\
\hline 0.5 & $\left|\delta \mathbf{r}_{Y Z}^{C} / \delta N\right|$ & 0.69 & 0.74 & 0.71 & 0.77 & 0.49 & 0.64 & 0.53 \\
\hline 1 & $\underline{\alpha},{ }^{\circ}$ & 75.5 & 75.3 & 76.7 & 74.9 & 74.0 & 75.1 & 74.2 \\
\hline 0.25 & $\left|\delta \mathbf{r}_{Y Z}^{C} / \delta N\right|$ & 0.80 & 0.82 & 0.76 & 0.85 & 0.54 & 0.72 & 0.58 \\
\hline 1 & $\underline{\alpha},{ }^{\circ}$ & 77.6 & 76.7 & 77.7 & 76.3 & 75.7 & 76.9 & 75.6 \\
\hline 0.5 & $\left|\delta \mathbf{r}_{Y Z}^{C} / \delta N\right|$ & 1.45 & 1.53 & 1.47 & 1.60 & 0.99 & 1.32 & 1.06 \\
\hline 0.75 & $\underline{\alpha},{ }^{\circ}$ & 83.7 & 84.3 & 86.1 & 83.7 & 83.8 & 84.1 & 84.4 \\
\hline 0.25 & $\left|\delta \mathbf{r}_{Y Z}^{C} / \delta N\right|$ & 1.32 & 1.32 & 1.23 & 1.37 & 0.85 & 1.16 & 0.90 \\
\hline 0.75 & $\underline{\alpha},{ }^{\circ}$ & 85.2 & 84.6 & 85.8 & 83.8 & 84.0 & 84.8 & 84.3 \\
\hline 0.25 & $\left|\delta \mathbf{r}_{Y Z}^{C} / \delta N\right|$ & 2.94 & 3.00 & 2.95 & 3.07 & 1.92 & 2.62 & 2.05 \\
\hline 0.5 & $\underline{\alpha},{ }^{\circ}$ & 90.6 & 89.2 & 88.8 & 88.8 & 89.1 & 89.8 & 88.7 \\
\hline Fit & $\left|\delta \mathbf{r}_{Y Z}^{C} / \delta N\right|$ & 0.71 & 0.74 & 0.69 & 0.77 & 0.49 & 0.64 & 0.52 \\
\hline & $\underline{\alpha},{ }^{\circ}$ & 76.3 & 75.7 & 77.0 & 75.4 & 74.5 & 75.7 & 74.5 \\
\hline
\end{tabular}

Примеч ани е. ${ }^{1}$ Расчет для пар точек $x_{1}$ и $x_{2}$ по (8). ${ }^{2}$ Расчет для всех точек Fit по (9).

Таблица 3. Компоненты $\delta \mathbf{F}_{Y Z} / \delta N$, определенные по данным $\delta \alpha / \delta N$ для трех пар ${ }^{1}$ точек с $x_{2}=1$ и по всем данным ${ }^{2} \delta \alpha(x) / \delta N$ измеренного профиля

\begin{tabular}{|c|c|c|c|c|c|c|c|c|}
\hline \multirow{2}{*}{$x_{1}$} & \multirow{2}{*}{ Компоненты } & \multicolumn{7}{|c|}{ Участки разного наклона на профиле высоты, рис. $3, b$} \\
\hline & & 1 & 2 & 3 & 4 & 5 & 6 & 7 \\
\hline \multirow{3}{*}{0.75} & $\left|\delta \mathbf{F}_{Y Z} / \delta N\right|, \mathrm{N} / \mathrm{m}$ & 76 & 55 & 58 & 63 & 30 & 47 & 35 \\
\hline & $\bar{\alpha},{ }^{\circ}$ & 129 & 111 & 121 & 114 & 53 & 111 & 39 \\
\hline & $\bar{x}_{1(\text { extr })}$, расчет $^{3}$ & 0.69 & 0.70 & 0.70 & 0.72 & 0.64 & 0.70 & 0.61 \\
\hline \multirow{3}{*}{0.5} & $\left|\delta \mathbf{F}_{Y Z} / \delta N\right|, \mathrm{N} / \mathrm{m}$ & 92 & 92 & 128 & 86 & 39 & 74 & 45 \\
\hline & $\bar{\alpha}, \stackrel{\circ}{ }$ & 134 & 132 & 142 & 128 & 111 & 140 & 116 \\
\hline & $\bar{x}_{1(\text { extr })}$, расчет $^{3}$ & 0.68 & 0.69 & 0.64 & 0.70 & 0.73 & 0.63 & 0.71 \\
\hline \multirow{3}{*}{0.25} & $\left|\delta \mathbf{F}_{Y Z} / \delta N\right|, \mathrm{N} / \mathrm{m}$ & 173 & 149 & 168 & 138 & 76 & 134 & 80 \\
\hline & $\bar{\alpha},{ }^{\circ}$ & 146 & 143 & 146 & 140 & 136 & 143 & 136 \\
\hline & $\bar{x}_{1(\text { extr })}$, расчет $^{3}$ & 0.61 & 0.64 & 0.61 & 0.66 & 0.68 & 0.64 & 0.68 \\
\hline \multirow{2}{*}{ Fit } & $\left|\delta \mathbf{F}_{Y Z} / \delta N\right|, \mathrm{N} / \mathrm{m}$ & 119 & 107 & 135 & 101 & 48 & 92 & 52 \\
\hline & $\bar{\alpha},{ }^{\circ}$ & 141 & 137 & 144 & 134 & 123 & 137 & 124 \\
\hline
\end{tabular}

Приме чан ие. ${ }^{1}$ Расчет по (8). ${ }^{2}$ Расчет по $(10) .{ }^{3}$ Расчет по методике разд. 2.2.

$\lambda=0.2$ и $\left.\bar{\alpha}=90^{\circ}\right)$. Согласно (5), $\bar{\alpha}=110^{\circ}$ переходит в $\underline{\alpha} \cong 74^{\circ}$. Это дает направление скольжения острия зонда „идеального кантилевера“ на горизонтальной поверхности, перемещающейся вертикально (трения нет, сила действует строго вертикально). Значения $\underline{\alpha}$ в табл. 2 можно трактовать следующим образом. Когда решетка поднимается или опускается, острие зонда на горизонтальном участке выступа двигается по траектории 
скольжения, а на левом и правом склонах выступа идет слегка выше или соответственно ниже этой траектории.

В каждой секции табл. 2, для 3-х пар с $x_{2}=1$ и Fit, величина $\left|\delta \mathbf{r}_{Y Z}^{C} / \delta N\right|<1$ и максимальна на 4-м участке. Уменьшение $\left|\delta \mathbf{r}_{Y Z}^{C} / \delta N\right|$ на вертикальных краях выступа можно связать с проскальзыванием зонда на них, см. работу [18] о корректировке сигналов кажущейся жесткости и деформации на склонах рельефа однородного образца. Без учета деформации образца отличие $\left|\delta \mathbf{r}_{Y Z}^{C} / \delta N\right|$ от единицы на 4-м участке определяется дифференциальной деформацией $\delta \mathbf{r}_{Y Z}^{T} / \delta N$ (см. $\mathbf{r}_{Y Z}^{T}$ на рис. 1).

Диапазоны углов $\bar{\alpha}$ вектора $\delta \mathbf{F}_{Y Z} / \delta N$ в табл. 3 и $\underline{\alpha}$ вектора $\delta \mathbf{r}_{Y Z}^{C} / \delta N$ в табл. 2 заметно отличаются. Отметим, что в обычном анализе кривых $\operatorname{DFL}(N)$ [21] сила действует вертикально, $\bar{\alpha}=110^{\circ},\left|\delta \mathbf{F}_{Y Z} / \delta N\right|=k_{C}=69 \mathrm{~N} / \mathrm{m}$, и получается результат, не зависящий от наклона образца и положения фокуса лазера на консоли.

Для всех 4-х случаев табл. 3 поведение $\bar{\alpha}$ на разных участках выступа качественно похоже. Наиболее ярко его демонстрируют данные пары $x_{1}=0.75$ и $x_{2}=1$, отображенные также семью стрелками на рис. 3, $b$. В сравнении с горизонтальным 4-м участком, на краях выступа растет боковая сила. Это согласуется с представлением, что острый зонд давит на плоский горизонтальный участок выступа вертикально, а острый край выступа давит на боковую поверхность зонда под углом.

На схеме кантилевера (рис. 3,b) у зонда почти вертикальная задняя (слева) и наклоненная передняя (справа) стороны. По инструментальному уширению вертикальных участков выступа решетки TGZ2 в профиле АСМ-сигнала высоты можно восстановить форму самого острия зонда [21]. Результат этой оценочной процедуры показан на схеме слева на рис. $3, b$ : кончик зонда выглядит затупившимся, сохранив асимметрию. Предположение, что такой зонд сильнее проскальзывает на правом, чем на левом краю выступа можно проверять по ослаблению взаимодействия. Амплитуда дифференциальной силы, длина стрелок на рис. $3, b$ на правом краю, действительно, заметно меньше, чем на 4-м горизонтальном участке и левом краю выступа. Однако максимальна она не на 4-м, а на 1-м участке, на левом краю.

На качественном уровне, $\left|\delta \mathbf{F}_{Y Z} / \delta N\right|$ можно смоделировать как АСМ-сигнал жесткости, см. уравнение (11a) в [18]. В [18] учитывался, в частности, наклон консоли и локальный рельеф образца, но явно не брался в расчет вклад геометрии острия зонда. Если пренебречь вкладом жесткости самого зонда, такое моделирование показывает максимум сигнала на левом и минимум на правом краю выступа. Для кантилевера, развернутого параллельно выступам решетки, предсказывается падение сигнала на обоих краях выступа относительно горизонтального участка независимо от вклада жесткости самого зонда. В такой связи мы полагаем, что наблюдаемое поведение амплитуды дифференциальной силы (рис. 3, $b$ и табл. 3) главным образом, связано с выбранным расположением кантилевера, и частично с вертикальной асимметрией формы зонда.

На рис. 3, $a$ кривая $0.5-1$, точно проходящая через точки с абсциссами $x_{1}=0.5$ и $x_{2}=1$, практически совпадает с кривой Fit, подгонкой модели ко всему экспериментальному профилю, хотя, как и Fit, не пересекает область разброса значений точки $x=0.75$. Кривая 0.75-1 идет немного ниже Fit, но пересекает области разброса значений точек $x=0.5$ и 0.25 . Кривая 0.25-1 плохо согласуется с Fit, не пересекает область разброса значений точек $x=0.75$ и касается такой области у точки $x=0.5$. Это типичная картина для всех семи участков (табл. 3 ).

Используя значения компонент $\delta \mathbf{F}_{Y Z} / \delta N$ с разными $x_{1}$, по методике разд. 2.2 были рассчитаны $\bar{x}_{1(\text { extr })}$ в табл. 3. (По соответствующим данным в табл. 2 получаются $\underline{x}_{1(\text { extr })}$, совпадающие с $\bar{x}_{1(\text { extr })}$ во втором знаке). В секциях с $x_{1}=0.75,0.5,0.25$ средние по семи участкам $\left\langle x_{1(\text { extr })}\right\rangle \cong 0.68,0.68,0.65$ соответственно. Использованное в измерениях значение $x_{1}=0.75$ ближе других к среднему расчетному 0.68 и к полусумме оценок, полученных в разд. 2.2, 0.68. Однако в табл. 3 данные секции с $x_{1}=0.5$, следующим значением по близости к 0.68 , наилучшим образом согласуются с данными секции Fit и, в частности, описывают весь экспериментальный профиль на рис. $3, a$.

В итоге, можно констатировать удовлетворительное согласие эксперимента по картированию компонент силы с результатами теоретического раздела. Отмеченные расхождения, как мы полагаем, связаны с неточностью значений $\sigma_{C_{1}} / C_{1}$ и $\sigma_{Y}$, примененных в расчетах $x_{1(\text { extr) }}$, и с не строгим выполнением в измерениях одного из условий (П3), $\sigma_{y_{1}}=\sigma_{y_{2}}$.

\section{Заключение}

Для картирования векторов силы взаимодействия зонд-образец и смещения „идеального кантилевера“ с помощью атомно-силового микроскопа со схемой оптического рычага достаточно определить углы изгиба в двух точках на консоли кантилевера и угол кручения в одной из них. В работе исследованы вопросы проведения и оптимизации таких измерений. Доказано существование двух особых точек на прямоугольной консоли, в совокупности сводящих к минимуму ошибки измерений трех пространственных компонент силы и вектора смещения. Положение одной особой точки рассчитывается как корень полинома четвертой степени, аналитические выражения коэффициентов которого приведены в табл. 1, особенности расчета изложены в разд. 2.2. Другая особая точка находится на пересечении плоскости консоли с осью зонда.

На образце калибровочной решетки TGZ2 проведен демонстрационный эксперимент по картированию пространственных компонент силы, действующей на 
АСМ-зонд. Полученные экспериментальные данные удовлетворительно согласуются с расчетами. Дальнейшее тестирование теории требует дополнительных измерений. Существенно проще такие эксперименты в новых квазистатических режимах ACM - гибридном, HybriD (НТ-МДТ СИ), PeakForce QNM (Bruker), fast force volume mapping (Asylum Research, США) — в основе работы которых лежат быстрые регистрация и анализ нагрузочно-разгрузочных зависимостей $\operatorname{DFL}(N)$.

Результаты работы представляются важными для развития наномеханических АСМ-исследований. Знание амплитуды и направления действующей силы необходимо для измерений методом испытаний на изгиб модуля Юнга наномостиков, при изучении свойств прикрепленных к зонду нанообъектов, в экспериментах с биологическими клетками. На определение вектора смещения кончика зонда нацелены исследования нанодоменов электрической поляризации методом силовой микроскопии пьезоотклика.

\section{Благодарности}

Авторы благодарят М. М. Халисова за помощь в подготовке работы.

\section{Финансирование работы}

Исследование выполнено при поддержке Российского научного фонда, грант № 19-13-00151. Точное решение задачи поиска минимума ошибок, представленное в Математическом приложении к статье, получено А.М. Минарским, СПбАУ РАН им. Ж.И. Алфёрова, в рамках государственного задания 0791-2020-0009 Министерства науки и высшего образования РФ.

\section{Конфликт интересов}

Авторы заявляют, что у них нет конфликта интересов.

\section{Приложение}

\section{3. Математическое приложение}

\section{1. Постановка задачи}

Запишем общее выражение для профиля угла изгиба консоли кантилевера $\alpha(x)$, под действием приложенной к острию зонда кантилевера и лежащей в плоскости $Y Z$ силы $\mathbf{F}_{Y Z}=\left(F_{Y}, F_{Z}\right)$, вызывающим смещение острия недеформируемого зонда „идеального кантилевера“ в этой плоскости вдоль вектора $\mathbf{r}_{Y Z}^{C}=\left(Y^{C}, Z^{C}\right)$ :

$$
\begin{aligned}
y & =\alpha(x)=\frac{6\left(\lambda F_{Y}+F_{Z}\right) x-3 F_{Z} x^{2}}{2 k_{C} l_{C}} \\
& =\frac{\left(6 \lambda Z^{C}-2 Y^{C}\right) x-\left(6 \lambda Z^{C}-3 Y^{C}\right) x^{2}}{l_{T}}=a x-b x^{2} .
\end{aligned}
$$

Использованы: $\lambda=l_{T} / l_{C}$ - отношение высоты зонда $l_{T}$ к длине консоли $l_{C}$, коэффициент изгибной жесткости консоли $k_{C}$, нормированная координата $x=Y / l_{C}$ фокуса лазера схемы ОР на консоли, $x \in[0 ; 1]$.

Коэффициенты $a$ и $b$ в (П1) однозначно определяются по измеренным $y_{1}$ и $y_{2}$ в двух разных точках консоли, $x_{1}$ и $x_{2}$ :

$$
\left\{\begin{array} { l } 
{ y _ { 1 } = a x _ { 1 } - b x _ { 1 } ^ { 2 } } \\
{ y _ { 2 } = a x _ { 2 } - b x _ { 2 } ^ { 2 } }
\end{array} \rightarrow \left\{\begin{array}{l}
a=\frac{1}{\left(x_{2}-x_{1}\right)}\left(x_{2} \frac{y_{1}}{x_{1}}-x_{1} \frac{y_{2}}{x_{2}}\right), \\
b=\frac{1}{\left(x_{2}-x_{1}\right)}\left(\frac{y_{1}}{x_{1}}-\frac{y_{2}}{x_{2}}\right) .
\end{array}\right.\right.
$$

Пусть ошибки измерений подчиняются в среднем следующим соотношениям:

$$
\begin{gathered}
\left\langle\left(\Delta x_{1}\right)^{2}\right\rangle=\left\langle\left(\Delta x_{2}\right)^{2}\right\rangle=\sigma_{x}^{2},\left\langle\left(\Delta y_{1}\right)^{2}\right\rangle=\left\langle\left(\Delta y_{2}\right)^{2}\right\rangle=\sigma_{y}^{2}, \\
\left\langle\Delta x_{1} \Delta x_{2}\right\rangle=\left\langle\Delta y_{1} \Delta y_{2}\right\rangle=\left\langle\Delta y_{1} \Delta x_{1}\right\rangle=\left\langle\Delta y_{1} \Delta x_{2}\right\rangle \\
=\left\langle\Delta x_{1} \Delta y_{2}\right\rangle=\left\langle\Delta x_{2} \Delta y_{2}\right\rangle=0
\end{gathered}
$$

Сформулируем задачу. Для заданных дисперсий $\sigma_{x}^{2}$ и $\sigma_{y}^{2}$ требуется найти $x_{1}, x_{2} \in[0 ; 1]$, обеспечивающие минимальные дисперсии $\sigma_{a}^{2}$ и $\sigma_{b}^{2}$ :

$$
\left\{\begin{array}{l}
\sigma_{a}^{2}=\left[\left(\frac{\partial a}{\partial x_{1}}\right)^{2}+\left(\frac{\partial a}{\partial x_{2}}\right)^{2}\right] \sigma_{x}^{2}+\left[\left(\frac{\partial a}{\partial y_{1}}\right)^{2}+\left(\frac{\partial a}{\partial y_{2}}\right)^{2}\right] \sigma_{y}^{2}, \\
\sigma_{b}^{2}=\left[\left(\frac{\partial b}{\partial x_{1}}\right)^{2}+\left(\frac{\partial b}{\partial x_{2}}\right)^{2}\right] \sigma_{x}^{2}+\left[\left(\frac{\partial b}{\partial y_{1}}\right)^{2}+\left(\frac{\partial b}{\partial y_{2}}\right)^{2}\right] \sigma_{y}^{2} .
\end{array}\right.
$$

Для общности будем минимизировать линейную комбинацию дисперсий

$$
M=\cos ^{2} \theta \sigma_{a}^{2}+\sin ^{2} \theta \sigma_{b}^{2}=m_{x}^{2} \sigma_{x}^{2}+m_{y}^{2} \sigma_{y}^{2} .
$$

Выражения для $m_{x}^{2}, m_{y}^{2}$ следуют из (П4). Параметр $\theta$ условный, вспомогательный, в зависимости от выбора его величины значимы: только $\sigma_{a}^{2}, \cos \theta=1$; только $\sigma_{b}^{2}$, $\sin \theta=1$; обе ошибки, $\cos \theta=\sin \theta$.

\section{2. Случай $\sigma_{x} \ll \sigma_{y}$}

Рассмотрим $m_{y}^{2}$, квадрат чувствительности к ошибке по $y$

$$
m_{y}^{2} \sigma_{y}^{2}=\cos ^{2} \theta \sigma_{a}^{2}+\sin ^{2} \theta \sigma_{b}^{2} .
$$

С учетом (П2), (П4),

$$
\left(\partial a / \partial y_{1}\right)^{2}+\left(\partial a / \partial y_{2}\right)^{2}=\left(x_{1}^{4}+x_{2}^{4}\right) /\left(x_{1}^{2} x_{2}^{2}\left(x_{2}-x_{1}\right)^{2}\right)
$$

и

$$
\left(\partial b / \partial y_{1}\right)^{2}+\left(\partial b / \partial y_{2}\right)^{2}=\left(x_{1}^{2}+x_{2}^{2}\right) /\left(x_{1}^{2} x_{2}^{2}\left(x_{2}-x_{1}\right)^{2}\right)
$$

получаем

$$
m_{y}^{2}=\frac{\cos ^{2} \theta\left(x_{1}^{4}+x_{2}^{4}\right)+\sin ^{2} \theta\left(x_{1}^{2}+x_{2}^{2}\right)}{x_{1}^{2} x_{2}^{2}\left(x_{2}-x_{1}\right)^{2}} .
$$


Чтобы исследовать на экстремум, введем новые переменные $2 d=x_{2}-x_{1}$ и $p^{2}=x_{1} x_{2}$, и перепишем (П6b):

$$
m_{y}^{2}=\cos ^{2} \theta\left(\frac{4 d^{2}}{p^{4}}+\frac{4}{p^{2}}+\frac{1}{2 d^{2}}\right)+\sin ^{2} \theta\left(\frac{1}{p^{4}}+\frac{1}{p^{2} d^{2}}\right) \text {. }
$$

Если $d \neq 0$, то $\partial\left(m_{y}^{2}\right) / \partial p<0$. Следовательно, для любого фиксированного $d, m_{y}^{2}$ убывает с ростом $p$. Поэтому для каждого $x_{2} \in[2 d ; 1]$, минимум $m_{y}^{2}$ достигается при наибольшем возможном $p^{2}=\left(x_{2}-2 d\right) x_{2}$, т.е. в точке $x_{2}=x_{2(\text { extr })}=1$. С учетом этого (Пбb) записывается как функция одной переменной $x_{1}=x$ :

$$
m_{y}^{2}=\frac{1+x^{2} \sin ^{2} \theta+x^{4} \cos ^{2} \theta}{x^{2}(1-x)^{2}} .
$$

Найдем еще одну координату экстремума

$$
\frac{\partial\left(m_{y}^{2}\right)}{\partial x}=0 \rightarrow x^{4} \cos ^{2} \theta+x^{3} \sin ^{2} \theta+2 x-1=0 .
$$

Корень уравнения (П7) слабо зависит от $\theta$ : $x_{1(\text { extr })} \in[0.453 ; 0.475], x_{1(\text { extr })} \cong 0.464$.

Таким образом, если существенна только ошибка измерений угла изгиба консоли, то наиболее точное определение вектора приложенной силы обеспечивается измерениями в точках $x_{2(\text { extr })}=1$ и $x_{1(\text { extr })} \cong 0.464$.

\section{3. Случай $\sigma_{y} \ll \sigma_{x}$}

Рассмотрим $m_{x}^{2}$, квадрат чувствительности к ошибке по $x$

$$
m_{x}^{2} \sigma_{x}^{2}=\cos ^{2} \theta m_{a x}^{2} \sigma_{x}^{2}+\sin ^{2} \theta m_{b x}^{2} \sigma_{x}^{2}
$$

Используя (П2), (П4) и (П8а), с учетом

$m_{a x}^{2}=\left(\frac{\partial a}{\partial x_{1}}\right)^{2}+\left(\frac{\partial a}{\partial x_{2}}\right)^{2}=\frac{x_{1}^{4}\left(2 b x_{2}-a\right)^{2}+x_{2}^{4}\left(2 b x_{1}-a\right)^{2}}{x_{1}^{2} x_{2}^{2}\left(x_{2}-x_{1}\right)^{2}}$

$m_{b x}^{2}=\left(\frac{\partial b}{\partial x_{1}}\right)^{2}+\left(\frac{\partial b}{\partial x_{2}}\right)^{2}=\frac{x_{1}^{2}\left(2 b x_{2}-a\right)^{2}+x_{2}^{2}\left(2 b x_{1}-a\right)^{2}}{x_{1}^{2} x_{2}^{2}\left(x_{2}-x_{1}\right)^{2}}$

получаем

$$
\begin{gathered}
\left(\cos ^{2} \theta x_{1}^{4}+\sin ^{2} \theta x_{1}^{2}\right)\left(2 b x_{2}-a\right)^{2} \\
m_{x}^{2}=\frac{+\left(\cos ^{2} \theta x_{2}^{4}+\sin ^{2} \theta x_{2}^{2}\right)\left(2 b x_{1}-a\right)^{2}}{x_{1}^{2} x_{2}^{2}\left(x_{2}-x_{1}\right)^{2}} .
\end{gathered}
$$

Ведем новые переменные $u$ и $v$ :

$$
\left\{\begin{array}{l}
x_{1}=\frac{a}{2 b} \frac{1}{u}=v x_{2}, \\
x_{2}=\frac{a}{2 b} \frac{1}{u v}
\end{array}\right.
$$

Считая $0 \leq x_{1} \leq x_{2} \leq 1, \quad$ имеем $\quad 0 \leq v \leq 1 \quad$ и $u \geq a /(2 b v)$. При подстановке (П9) в (П8d) получаем, учитывая (П8а):

$$
\begin{gathered}
m_{a x}^{2}=4 b^{2}\left[\frac{1+v^{4}}{(1-v)^{2}}\left(u-u_{1}\right)^{2}+\frac{v^{2}}{1+v^{4}}\right], u_{1}=\frac{1+v^{3}}{1+v^{4}}, \\
m_{b x}^{2}=\frac{16 b^{2}}{a^{2}}\left[\frac{v^{2}\left(1+v^{2}\right)}{(1-v)^{2}}\left(u-u_{2}\right)^{2} u^{2}+\frac{v^{2}}{1+v^{4}} u^{2}\right], \\
u_{2}=\frac{1+v}{1+v^{2}} .
\end{gathered}
$$

Из (П10) и (П11) и $0 \leq v \leq 1$ также получаем

$$
u_{2}(v)-u_{1}(v)=\frac{(1-v)\left(1-v^{2}\right) v}{\left(1+v^{2}\right)\left(1+v^{4}\right)} \geq 0 .
$$

Можно видеть, что $\partial\left(m_{a x}^{2}\right) / \partial u \geq 0$ и $\partial\left(m_{b x}^{2}\right) / \partial u \geq 0$ пока $u \geq u_{2}(v) \geq u_{1}(v)$. Действительно, в квадратных скобках (П10) стоит выражение для параболы, растущей при $u \geq u_{1}(v)$. В квадратных скобках (П11) заключено более сложное выражение. Но, так как с увеличением $\mathrm{u}$ его левое слагаемое растет при $u \geq u_{2}(v)$, а правое всегда растет, то при $u \geq u_{2}(v)$ растет и их сумма.

Как следствие, если

$$
u_{2}(v) \geq \frac{a}{2 b} \frac{1}{v}, \text { что эквивалентно } \frac{v+v^{2}}{1+v^{2}} \leq \frac{a}{2 b},
$$

то $m_{a x}^{2}$ и $m_{b x}^{2}$ минимальны на кривой $u=a /(2 b v)$, т. е. $x_{2}=x_{2(\text { extr })}=1 \quad$ (см. (П9)). Поскольку $v \in[0 ; 1]$, (П13) выполняется всегда, когда $a \geq 2 b>0$.

Пусть теперь $0<a<2 b$ и рассмотрим сначала случай $m_{x}^{2}=m_{a x}^{2}(\theta=0)$.

Для каждого $v=\mathrm{const}$ из области II на рис. 4, $v_{1} \leq v \leq 1$, выражение (П10) минимально в точке кривой $u=u_{1}(v)$. На этой кривой $m_{a x}^{2}=4 b^{2} v^{2} /\left(1+v^{4}\right)$ зависит только от $v$ и минимален в точке пересечения с гиперболой $u=a /(2 b v)$. Значение $v=v_{1}$ находится как корень уравнения $u_{1}(v)=a /(2 b v)$, или

$$
\frac{v+v^{4}}{1+v^{4}}=\frac{a}{2 b} \text {. }
$$

В области I на рис. 4, $0 \leq v \leq v_{1}$, для каждого $v=$ const минимум $m_{a x}^{2}$ достигается сразу же на гиперболе.

В итоге, для всех допустимых значений $u$ и $v, m_{a x}^{2}$ минимален на этой гиперболе (см. также подпись к рис. 4 и соотношения (П9)), т.е. на границе, соответствующей $x_{2}=x_{2(\text { extr })}=1$.

Итак, если $m_{x}^{2}=m_{a x}^{2}$, то минимум квадрата чувствительности по $x$ лежит в области $x_{1} \leq v_{1}, x_{2}=1$. Подставляя в (П10) $x_{1}=v$ и $x_{2}=1$, получаем, что другая координата минимума $m_{a x}^{2}, x_{1}=x_{1(\text { extr })}$, находится из минимизации по $v$ выражения

$$
m_{a x}^{2}=\frac{1}{v^{2}(1-v)^{2}}\left[v^{4}(2 b-a)^{2}+(2 b v-a)^{2}\right] .
$$




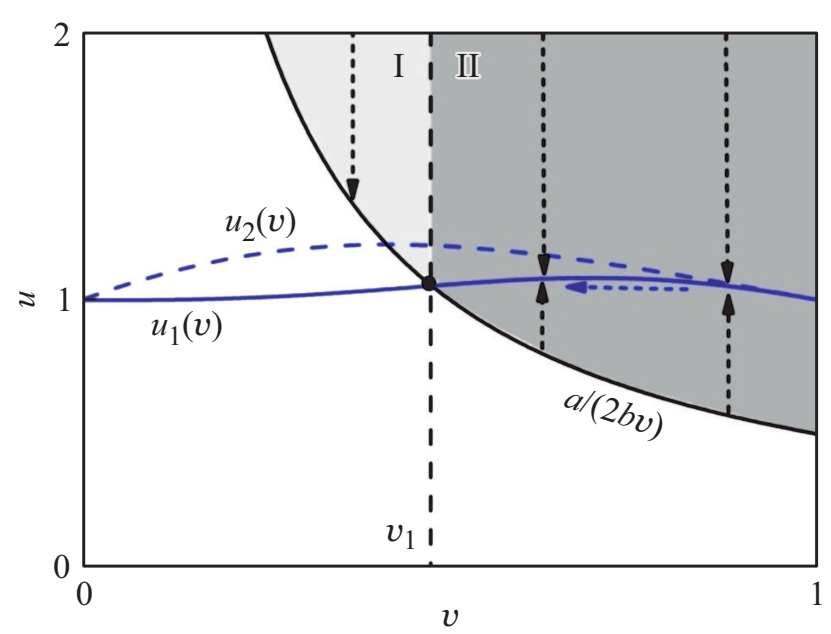

Рис. 4. Поиск минимума $m_{a x}^{2}$, (П9). Гипербола $u=a /(2 b v)$, сплошная черная кривая, соответствующая $x_{2}=1$, ограничивает снизу области I и II допустимых значений $u$. Для каждого $v=$ const вертикальные, пунктирные стрелки указывают направление изменений переменной $u$, уменьшающих $m_{a x}^{2}$. В области $\mathrm{I}, 0 \leq v<v_{1}, m_{a x}^{2}$ достигает минимума на гиперболе. Для каждого $v=$ const в области II, $v_{1} \leq v \leq 1, m_{a x}^{2}$ наименьший в соответствующей точке на сплошной кривой $u=u_{1}(v)$. В свою очередь, вдоль этой кривой $m_{a x}^{2}$ убывает с падением $v$, см. пунктирную стрелку под ней, и достигает минимума в точке пересечения с гиперболой, $v=v_{1}$.

Или, дифференцируя (П15), из корня уравнения

$$
\begin{gathered}
P_{a}(v)=(2 b-a)^{2} v^{4}+4 b^{2} v^{3}-6 a b v^{2} \\
+\left(2 a b+2 a^{2}\right) v-a^{2}=0 . \\
P_{a}(0)=-a^{2}<0, P_{a}(a / 2 b)=(a(2 b-a) / 2 b)^{2}>0
\end{gathered}
$$

и

$$
P_{a}(1)=2(2 b-a)^{2}>0,
$$

и корни всегда существуют. Они зависят от $a / b$, и следует выбирать минимальный корень, который для $0<a<2 b$ будет меньше $v_{1}$, определяемого уравнением (П14). Добавим также, что $x_{1} \leq v_{1}<a / 2 b$, если $a / 2 b<1$.

Перейдем к случаю $m_{x}^{2}=m_{b x}^{2}(\theta=\pi / 2)$.

Запишем (П11) по-другому:

$$
\begin{gathered}
m_{b x}^{2}=\frac{16 b^{2}}{a^{2}}\left[c\left(u-u_{2}\right)^{2} u^{2}+d u^{2}\right], \\
u_{2}=\frac{1+v}{1+v^{2}}, c=\frac{v^{2}\left(1+v^{2}\right)}{(1-v)^{2}}, d=\frac{v^{2}}{1+v^{4}} .
\end{gathered}
$$

Дифференцируя (П17) по $u$, получим условия для экстремумов $m_{b x}^{2}$ :

$$
\left(2 c u^{2}-3 c u_{2} u+c u_{2}^{2}+d\right) u=0 .
$$

Три корня (П18) несложно вычислить:

$$
\begin{aligned}
u=0, u_{ \pm} & =\frac{3 u_{2}}{4} \pm \sqrt{\left(\frac{u_{2}}{4}\right)^{2}-\frac{d}{2 c}} \\
& =\frac{3(1+v) \pm \sqrt{(1+v)^{2}-8(1-v)^{2}}}{4\left(1+v^{2}\right)} .
\end{aligned}
$$

На рис. 5 анализируется, где достигается минимум $m_{b x}^{2}$. В двух частных случаях на рис. $5, m_{b x}^{2}$ минимален в области I на гиперболах $u=0.16 / v$ и соответственно $u=0.62 / v$. Для каждого $v=$ const в области II на обоих рисунках, $m_{b x}^{2}$ наименьший на кривой $u=u_{+}$.

Докажем, что на кривой $u=u_{+}$:

$$
\frac{d\left(m_{b x}^{2}\right)}{d v}>0
$$

Если это верно, то на этой кривой значения $m_{b x}^{2}$ наименьшие: в точке ветвления $v_{2}$, где $u_{-}=u_{+}$(рис. $5, a$ ), в точке $v_{3}$, пересечения с кривой $u=u_{+}=0.62 / v$ (рис. $5, b$ ). В первом случае $m_{b x}^{2}$ продолжает убывать при движении вниз вдоль стрелки из точки ветвления в точку пересечения вертикальной линии $v=v_{2}$ с кривой $u=0.16 / v$, где достигается минимум $m_{b x}^{2}$ и выполняется условие $x_{2}=1$. Во втором случае минимум достигается сразу на пересечении с гиперболой, что автоматически соответствует условию $x_{2}=1$.

Согласно (П18), $d\left(m_{b x}^{2}\right) /\left.d u\right|_{u=u_{+}}=0$. Следовательно:

$$
\begin{aligned}
& \left.\frac{d\left(m_{b x}^{2}\right)}{d v}\right|_{u=u_{+}}=\frac{\partial\left(m_{b x}^{2}\right)}{\partial v}+\frac{\partial\left(m_{b x}^{2}\right)}{\partial u} \frac{d u_{+}}{d v}=\frac{\partial\left(m_{b x}^{2}\right)}{\partial v} \\
& =u_{+}^{2}\left[\frac{\partial c}{\partial v}\left(u_{2}-u_{+}\right)^{2}+2 c\left(u_{2}-u_{+}\right) \frac{\partial u_{2}}{\partial v}+\frac{\partial d}{\partial v}\right] .
\end{aligned}
$$

Для (П19)

$$
\begin{aligned}
u_{2}-u_{+} & =u_{2} / 4-\sqrt{\left(u_{2} / 4\right)^{2}-d / 2 c} \\
& =(d / 2 c) /\left(u_{2} / 4+\sqrt{\left(u_{2} / 4\right)^{2}-d / 2 c}\right) .
\end{aligned}
$$

Пусть

$$
\varepsilon u_{2} / 2=u_{2} / 4+\sqrt{\left(u_{2} / 4\right)^{2}-d / 2 c}, 1 / 2 \leq \varepsilon<1 .
$$

Тогда

$$
(1-\varepsilon) u_{2} / 2=u_{2} / 4-\sqrt{\left(u_{2} / 4\right)^{2}-d / 2 c} .
$$

Следовательно,

$$
\varepsilon(1-\varepsilon)\left(u_{2} / 2\right)^{2}=d / 2 c .
$$

Или с учетом (П17):

$$
\begin{gathered}
\varepsilon(1-\varepsilon)=\frac{2 d}{c u_{2}^{2}}=\frac{2(1-v)^{2}}{(1+v)^{2}}, \\
u_{2}-u_{+}=\frac{d}{\varepsilon c u_{2}} .
\end{gathered}
$$



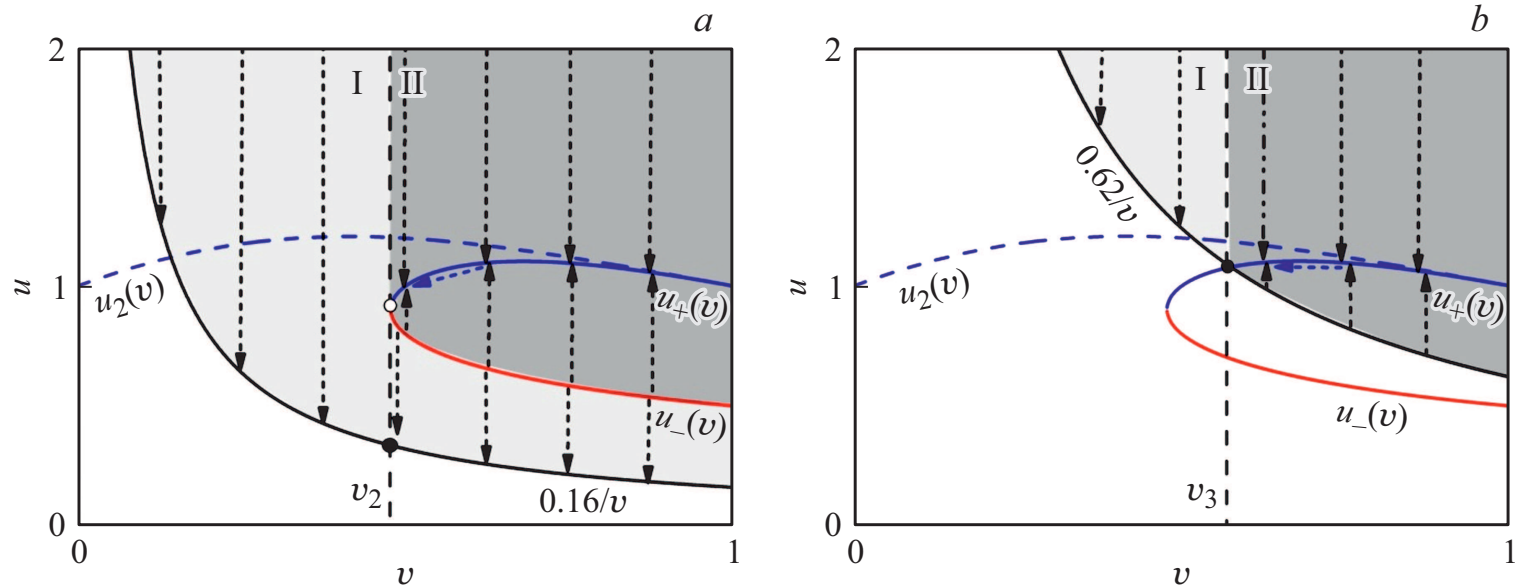

Рис. 5. Поиск минимума $m_{b x}^{2}$. Два частных случая. $a$ - гипербола, ограничивающая снизу область допустимых значений $u$, не пересекается с кривыми $u_{+}(v)$ и $u_{-}(v) . b-$ гипербола пересекается с кривой $u_{+}(v)$. См. также пояснения и подпись к рис. 4.

Подставляя (П22) и (П23) в (П21), получаем

$$
\begin{gathered}
\frac{\partial\left(m_{b x}^{2}\right)}{\partial v}=u_{+}^{2}\left[\left(\frac{d}{\varepsilon c u_{2}}\right)^{2} \frac{\partial c}{\partial v}+\frac{2 d}{\varepsilon u_{2}} \frac{\partial u_{2}}{\partial v}+\frac{\partial d}{\partial v}\right] \\
=\frac{u_{+}^{2} d}{\varepsilon}\left[(1-\varepsilon) \frac{\partial \ln (c)}{\partial v}+\varepsilon \frac{\partial \ln (d)}{\partial v}+2 \frac{\partial \ln \left(u_{2}\right)}{\partial v}\right] .
\end{gathered}
$$

С учетом (П17)

$$
\begin{aligned}
\frac{\varepsilon}{u_{+}^{2} d} \frac{\partial\left(m_{b x}^{2}\right)}{\partial v} & =(1-\varepsilon)\left[\frac{1}{1-v}+\frac{1}{v}+\frac{v}{1+v^{2}}\right] \\
& +\varepsilon\left[\frac{2}{v}+\frac{2 v}{1+v^{2}}\right]+\frac{2}{1+v}-\frac{4 v}{1+v^{2}} \\
& =\frac{1-\varepsilon}{1-v}+\frac{1+\varepsilon}{v}+\frac{2}{1+v}-\frac{(3-\varepsilon) v}{1+v^{2}} \\
& \geq \frac{1+\varepsilon}{v}+\frac{2}{1+v}-\frac{2 v}{1+v^{2}} \geq \frac{1+\varepsilon}{v}>0 .
\end{aligned}
$$

Итак: доказано, что $d\left(m_{b x}^{2}\right) / d v>0$ на кривой $u=u_{+}$и, в частности, убывание $m_{b x}^{2}$ при движении в направлении стрелок под этой кривой, показанных на рис. 5.

Таким образом, $m_{b x}^{2}$ достигает минимума при условии, что $x_{1}=v$ и $x_{2}=1$. Подставляя условие в (П8b), получаем

$$
m_{b x}^{2}=\frac{(2 b-a)^{2}}{(1-v)^{2}}+\frac{(2 b v-a)^{2}}{v^{2}(1-v)^{2}} .
$$

Минимум (П25) находится из следующего уравнения:

$$
\begin{aligned}
P_{b}(v)= & \left((2 b-a)^{2}+4 b^{2}\right) v^{3}-6 a b v^{2} \\
& +\left(2 a b+2 a^{2}\right) v-a^{2}=0 . \\
P_{b}(0)= & -a^{2}<0 \text { и } P_{b}(1)=2(2 b-a)^{2}>0,
\end{aligned}
$$

поэтому уравнение (П26), как и (П16), имеет один корень на интервале $0 \leq v \leq 1$.
Если $\quad b \ll a \quad\left(\bar{\alpha} \rightarrow \pi n, \quad F_{Z} \rightarrow 0 \quad\right.$ (рис. $\left.2, a\right)$; $\underline{\alpha} \rightarrow \operatorname{arctg}(1 / 2 \lambda)+\pi n, Y^{C} \rightarrow 2 \lambda Z^{C}$ (рис. $\left.\left.2, b\right)\right)$, уравнения (П16) и (П26) переходят в

$$
\left\{\begin{array}{l}
P_{a}(v) \rightarrow v^{4}+2 v-1=0 \\
P_{b}(v) \rightarrow v^{3}+2 v-1=0
\end{array}\right.
$$

Отметим, что в этом пределе $m_{x}^{2}$ и $m_{y}^{2}$ минимизируются в одной и той же точке.

Если $\quad a \ll b \quad\left(\bar{\alpha} \rightarrow-\operatorname{arctg} \lambda+\pi n, \quad \lambda F_{Y} \rightarrow-F_{Z}\right.$ (рис. 2, $a) ; \underline{\alpha} \rightarrow \operatorname{arctg}(1 / 3 \lambda)+\pi n, Y^{C} \rightarrow 3 \lambda Z^{C}$ (рис. 2,b)), уравнения (П16) и (П26) переходят в

$$
\left\{\begin{array}{l}
P_{a}(v) \rightarrow v=0 \\
P_{b}(v) \rightarrow v=0 .
\end{array}\right.
$$

Для произвольных значений $a$ и $b$ уравнения для минимизации $m_{a x}^{2}$ и $m_{b x}^{2}$ сводятся к

$$
\left\{\begin{aligned}
p_{a}(v)= & \frac{P_{a}(v)}{4 b^{2}}=(1-\delta)^{2} v^{4}+v^{3}-3 \delta v^{2} \\
& +\delta(1+2 \delta) v-\delta^{2}=0, \\
p_{b}(v)= & \frac{P_{b}(v)}{4 b^{2}}=\left((1-\delta)^{2}+1\right) v^{3}-3 \delta v^{2} \\
& +\delta(1+2 \delta) v-\delta^{2}=0,
\end{aligned}\right.
$$

где $\delta=a / 2 b, x_{1}=v$.

Для минимизации $m_{x}^{2}=\cos ^{2} \theta m_{a x}^{2}+\sin ^{2} \theta m_{b x}^{2}$ нужно искать корень уравнения

$$
\cos ^{2} \theta p_{a}(v)+\sin ^{2} \theta p_{b}(v)=0 .
$$

Выпишем также разложение решения (П29) по параметру $\delta=a / 2 b$ :

$$
\left\{\begin{array}{l}
p_{a}(v)=0, \quad v=\delta-\delta^{3}+\delta^{4} \\
p_{b}(v)=0, \quad v=\delta-\delta^{2}+4 \delta^{3}-18 \delta^{4} .
\end{array}\right.
$$


Минимизация относительных ошибок.

Минимизируем $\left(\sigma_{a} / a\right)^{2}+\left(\sigma_{a} / b\right)^{2}=\eta_{x}^{2} \sigma_{x}^{2}, \quad$ где $\eta_{x}^{2}=m_{a x}^{2} / a^{2}+m_{b x}^{2} / b^{2}$. С учетом $x=v$ и $\delta=a / 2 b$, запишем условие минимума относительной ошибки $\eta_{x}$ :

$$
\frac{p_{a}(v)}{a^{2}}+\frac{p_{b}(v)}{b^{2}}=0
$$

Или

$$
\begin{array}{r}
(1-\delta)^{2} v^{4}+\left(1+8 \delta-8 \delta^{2}+4 \delta^{3}\right) v^{3}-3(1+4 \delta) v^{2} \\
+\delta(1+2 \delta)(1+4 \delta) v-\delta^{2}(1+4 \delta)=0
\end{array}
$$

Величины $a$ и $b$ разных знаков.

Изложенный выше формальный ход доказательств применим для случаев одновременно отрицательных либо одновременно положительных $a$ и $b$. Рассмотрим ситуацию разных знаков $a$ и $b$ (подразумевается, что $\left.x_{1}, x_{2} \in[0 ; 1]\right)$.

Подстановка $x_{1}=v x_{2}=|a / 2 b| / u$, уточняющая (П9), дает вместо (П10), (П11) и (П17) выражения, отличающиеся только заменой знака при $u_{1}$ и $u_{2}$ :

$$
\begin{gathered}
m_{a x}^{2} \sim c_{1}\left(u+u_{1}\right)^{2}+d_{1}, \\
m_{b x}^{2} \sim\left(c_{2}\left(u+u_{2}\right)^{2}+d_{2}\right) u^{2},
\end{gathered}
$$

с теми же зависимостями от $v$ для $c_{1}, c_{2}, d_{1}, d_{2}, u_{1}$ и $u_{2}$, что и в (П10) и (П17). Выражения (П34) и (П35), как функции $u$, всегда убывают в области положительных $u$. Следовательно, при минимизации $m_{x}^{2}$ всегда достигается и ситуация $u=|a / 2 b| / v$, т. е. $x_{2(\text { extr })}=1$. Поэтому из (П34) и (П35), как функций $x_{1}=v$, после дифференцирования получатся те же уравнения, что (П16) и (П26), позволяющие найти искомые корни v на отрезке $[0 ; 1]$. Соотношения (П29) и (П33) также выполняются (только теперь $\delta \leq 0)$. Предельные соотношения (П27) и (П28) соблюдаются, а разложение (П31) не актуально, так как в рассматриваемом случае соответствует отрицательному $x_{1}=v$. Положительные значения $x_{1}=v$ даются следующими разложениями по $\delta(\delta<0,|\delta| \ll 1)$ :

$$
\begin{cases}p_{a}(v)=0: & v \cong \sqrt{|\delta|}-|\delta|, \\ p_{b}(v)=0: & v \cong \sqrt{\frac{|\delta|}{2}}-\frac{|\delta|}{2 \sqrt{2}} .\end{cases}
$$

Случай $a=2 b \quad\left(\bar{\alpha} \rightarrow \pi(2 n+1) / 2, \quad F_{Y}=0 \quad\right.$ (рис. $\left.2, a\right)$; $\underline{\alpha} \rightarrow \operatorname{arctg}(2 / 3 \lambda)+\pi n, 2 Y^{C} \rightarrow 3 \lambda Z^{C}$ (рис. $\left.2, b\right)$ ).

Подставив $\delta=1$ в (П29), получим: $p_{a}(v)=p_{b}(v)=$ $=(1-v)^{3}$, т.е. $x_{1}=x_{2}=1$. В том, что такое условие соответствует минимуму $m_{a x}^{2}$ и $m_{b x}^{2}$, можно убедиться, подставив в (П10) и (П11) связь $u=1 / v$, следующую из (П9) для $x_{2}=1$ и $a=2 b$. Тогда получим выражения

$$
\left\{\begin{array}{l}
m_{a x}^{2}=\frac{a^{2}}{v^{2}}, \\
m_{b x}^{2}=\frac{2 a^{2}}{1+v^{2}},
\end{array}\right.
$$

которые, действительно, имеют минимум при $v=1$, т. е. $x_{1}=x_{2}=1$.

Этот результат означает, что точный оптимум по $x_{1}$ и $x_{2}$ нельзя найти без учета вклада $m_{y}^{2}$.

\section{4. Общий случай}

Рассмотрим общий случай и, см. также (П5), минимизируем

$$
m_{x}^{2} \sigma_{x}^{2}+m_{y}^{2} \sigma_{y}^{2}
$$

где $m_{x}^{2}$ берется из (П8а), (П8b) и (П8с), $m_{y}^{2}$ из (П6b) и произведена подстановка $x_{1}=v$ и $x_{2}=1$. Если установить связь

$$
\sigma_{y}=k \sigma_{x}, \quad k>0,
$$

то придется минимизировать по $v$ следующее выражение:

$$
\frac{\left(\cos ^{2} \theta v^{2}+\sin ^{2} \theta\right)\left(k^{2}+(2 b-a)^{2}\right) v^{2}+(2 b v-a)^{2}+k^{2}}{v^{2}(1-v)^{2}} .
$$

Вклады $m_{y}^{2}$ и $m_{x}^{2}$ преобладают в зависимости от соотношения между $k$ и $|2 b-a|$. Соответственно условия минимума близки либо к $x_{1(\text { extr) }} \cong 0.464$ (большие $k$ ), либо (малые $k$ ) к корням уравнений (П16) или (П26).

Минимум (П40), $x_{1}=v$, находится в области $0<v<1$ как корень уравнения

$$
\begin{aligned}
P= & \cos ^{2} \theta\left[k^{2}\left(v^{4}+2 v-1\right)+P_{a}(v)\right] \\
& +\sin ^{2} \theta\left[k^{2}\left(v^{3}+2 v-1\right)+P_{b}(v)\right]=0,
\end{aligned}
$$

где $P_{a}(v)$ и $P_{b}(v)$ берутся из (П16) и (П26).

Когда $a=2 b$, то $P_{a}(v)=P_{b}(v)=4 b^{2}(1-v)^{3}=a^{2}(1-v)^{3}$, и (П41) сводится к

$$
\left\{\begin{array}{l}
\cos \theta=1, \min \left(m_{a}^{2}\right): k^{2}\left(v^{4}+2 v-1\right)+a^{2}(1-v)^{3}=0, \\
\sin \theta=1, \min \left(m_{b}^{2}\right): k^{2}\left(v^{3}+2 v-1\right)+a^{2}(1-v)^{3}=0 .
\end{array}\right.
$$

Корни (П42) зависят только от $k / a(\delta=1)$.

Случай $\delta=1$ и произвольного $\theta$ описывается уравнением

$$
k^{2}\left(\cos ^{2} \theta v^{4}+\sin ^{2} \theta v^{3}+2 v-1\right)+a^{2}(1-v)^{3}=0,
$$

корни которого слабо зависят от $\theta$.

Наиболее общим из уравнений (П41)-(П43) является (П41), его корень зависит от $\delta=a / 2 b, k / a$ и $\theta$.

Условие равных относительных чувствительностей, см. (П32), означает

$$
\cos ^{2} \theta=\frac{b^{2}}{a^{2}+b^{2}}, \quad \sin ^{2} \theta=\frac{a^{2}}{a^{2}+b^{2}} .
$$

(П44) можно прямо использовать в (П41), решение будет зависеть от $a / 2 b$ и $k / a$. 
В частности, для $a=2 b$ (П44) переходит в $\cos ^{2} \theta=1 / 5$ и $\sin ^{2} \theta=4 / 5$. Подстановка этого в (П32) для $k / a, k / b \ll 1$ дает

$$
x_{1}=v \cong 1-\sqrt[3]{2 k^{2} / a^{2}}=1-\sqrt[3]{k^{2} / 2 b^{2}} .
$$

Для $k=a=2 b,(П 42)$ имеет следующие приближенные корни

$$
\begin{cases}\min \left(m_{a}^{2}\right): & x_{1} \cong 0.52, \\ \min \left(m_{b}^{2}\right): & x_{1} \cong 0.50 .\end{cases}
$$

В заключение приведем выражения для $P$ в (П41) в общем случае и в частных случаях, когда $a \ll b$ либо $b \ll a$ :

$$
\begin{gathered}
P=\cos ^{2} \theta\left(k^{2}+(2 b-a)^{2}\right) v^{4}+\left(\sin ^{2} \theta\left(k^{2}+(2 b-a)^{2}\right)\right. \\
\left.+4 b^{2}\right) v^{3}-6 a b v^{2}+2\left(k^{2}+a b+a^{2}\right) v-\left(k^{2}+a^{2}\right), \\
\frac{\left.P\right|_{b a \ll b}}{4 b^{2}} \rightarrow \cos ^{2} \theta\left[\frac{k^{2}}{4 b^{2}}\left(v^{4}+2 v-1\right)+v^{4}+v^{3}\right] \\
\quad+\sin ^{2} \theta\left[\frac{k^{2}}{4 b^{2}}\left(v^{3}+2 v-1\right)+2 v^{3}\right], \\
\frac{\left.P\right|_{b \ll a}}{\left(k^{2}+a^{2}\right)} \rightarrow\left(\cos ^{2} \theta v+\sin ^{2} \theta\right) v^{3}+2 v-1 .
\end{gathered}
$$

Для $k / b \gg 1$ корень $\left(\right.$ П47b) $v=x_{1 \text { (extr) }} \cong 0.464$, если $k / b \ll 1$, то $v \cong 0$. Корень $(П 47 \mathrm{c})$ не зависит от $k / a$ и всегда близок к $v=x_{1(\text { extr })} \cong 0.464$.

\section{5. Расчет вспомогательного параметра $\theta$}

Значения вспомогательного параметра $\theta$ находятся с помощью (2), минимизируя $\sigma_{F_{Y}}^{2}+\sigma_{F_{Z}}^{2}$ или $\sigma_{Y^{C}}^{2}+\sigma_{Z^{C}}^{2}$, с учетом взаимной независимости ошибок определения $a$ и $b$. Для примененного в эксперименте кантилевера с $\lambda=0.2$ приведем выкладки расчета $\bar{\theta}$ :

$$
\begin{gathered}
\left\{\begin{array} { l } 
{ F _ { Y } = \frac { k _ { C } l _ { C } } { 3 \lambda } ( \overline { a } - 2 \overline { b } ) , } \\
{ F _ { Z } = \frac { 2 k _ { C } l _ { C } } { 3 } \overline { b } }
\end{array} \rightarrow \left\{\begin{array}{l}
\sigma_{F_{Y}}^{2}=\frac{k_{C}^{2} l_{C}^{2}}{9 \lambda^{2}}\left(\sigma_{\bar{a}}^{2}+4 \sigma_{\bar{b}}^{2}\right), \\
\sigma_{F_{Z}}^{2}=\frac{4 k_{C}^{2} l_{C}^{2}}{9} \sigma_{\bar{b}}^{2}
\end{array}\right.\right. \\
\sigma_{F_{Y}}^{2}+\sigma_{F_{Z}}^{2}=\frac{k_{C}^{2} l_{C}^{2}}{9 \lambda^{2}}\left(\sigma_{\bar{a}}^{2}+4\left(1+\lambda^{2}\right) \sigma_{\bar{b}}^{2}\right) \rightarrow \cos ^{2} \bar{\theta} \cong 0.19, \\
\sin ^{2} \bar{\theta} \cong 0.81
\end{gathered}
$$

а также $\underline{\theta}$ :

$$
\begin{gathered}
\left\{\begin{array} { c } 
{ Y ^ { C } = \lambda l _ { C } ( \underline { a } - \underline { b } ) , } \\
{ Z ^ { C } = \frac { l _ { C } } { 6 } ( 3 \underline { a } - 2 \underline { b } ) }
\end{array} \rightarrow \left\{\begin{array}{l}
\sigma_{Y^{C}}^{2}=\lambda^{2} l_{C}^{2}\left(\sigma_{\underline{a}}^{2}+\sigma_{\underline{b}}^{2}\right), \\
\sigma_{Z^{C}}^{2}=\frac{l_{C}^{2}}{36}\left(9 \sigma_{\underline{a}}^{2}+4 \sigma_{\underline{b}}^{2}\right)
\end{array}\right.\right. \\
\sigma_{Y^{C}}^{2}+\sigma_{Z^{C}}^{2}=l_{C}^{2}\left(\left(\lambda^{2}+1 / 4\right) \sigma_{\underline{a}}^{2}+\left(\lambda^{2}+1 / 9\right) \sigma_{\underline{b}}^{2}\right) \\
\rightarrow \cos ^{2} \underline{\theta} \cong 0.66, \quad \sin ^{2} \underline{\theta} \cong 0.34 .
\end{gathered}
$$

\section{Список литературы}

[1] G. Binnig, C.F. Quate, C. Gerber. Phys. Rev. Lett., 56 (9), 930 (1986). DOI: https://doi.org/10.1103/PhysRevLett.56.930

[2] Bhushan B. (Ed.) Nanotribology and Nanomechanics. An Introduction (Springer-Verlag, Berlin-Heidelberg, 2005)

[3] G. Dai, K. Hahm, F. Scholze, M.-A. Henn, H. Gross, J. Fluegge, H. Bosse. Meas. Sci. Technol., 25, 044002 (2014). DOI: $10.1088 / 0957-0233 / 25 / 4 / 044002$

[4] A. Bolopion, H. Xie, D.S. Haliyo, S. Régnier. IEEE/ASME Transactions on Mechatronics $17(1), 116 \quad$ (2012). DOI: 10.1109/TMECH.2010.2090892

[5] И.А. Няпшаев, А.В. Анкудинов, А.В. Стовпяга, Е.Ю. Трофимова, М.Ю. Еропкин. ЖТФ, 82 (10), 109 (2012).

[6] E. Soergel. J. Phys. D. Appl. Phys., 44, 464003 (2011). https://doi.org/10.1088/0022-3727/44/46/464003

[7] S. Fujisawa, M. Ohta, T. Konishi, Ya. Sugawara, S. Morita. Rev. Sci. Instrum., 65 (3), 644 (1994). DOI: https://doi.org/10.1063/1.1145131

[8] H. Kawakatsu, H. Bleuler, T. Saito, K. Hiroshi. Jpn. J. Appl. Phys., 34Pt1 (6B), 3400 (1995). DOI: https://doi.org/10.1143/JJAP.34.3400

[9] A. Labuda, R. Proksch. Appl. Phys. Lett., 106 (25), 253103 (2015). DOI: https://doi.org/10.1063/1.4922210

[10] Электронный ресурс. Режим доступа: https://afm.oxinst. com/assets/uploads/products/asylum/documents/Cypher-IDSOption-DS-March2018.pdf

[11] S. Alexander, L. Hellemans, O. Marti, J. Schneir, V. Elings, P.K.J. Hansma. J. Appl. Phys., 65 (1), 164 (1989). DOI: https://doi.org/10.1063/1.342563

[12] D. Rugar, H.J. Mamin, P. Guethner. Appl. Phys. Lett., 55 (25), 2588 (1989). DOI: https://doi.org/10.1063/1.101987

[13] R.S.M. Mrinalini, R. Sriramshankar, G.R. Jayanth. IEEE/ASME Transactions on Mechatronics, 20 (5), 2184 (2015). DOI: 10.1109/TMECH.2014.2366794

[14] R. Proksch, T.E. Schaffer, J.P. Cleveland, R.C. Callahan, M.B. Viani. Nanotechnology, 15 (9), 1344 (2004). DOI: $10.1088 / 0957-4484 / 15 / 9 / 039$

[15] A.V. Ankudinov. Nanosystems: Phys., Chem., Mathemat., $10(6), 642$ (2019). DOI: $10.17586 / 2220-8054-2019-10-6-642-653$

[16] D. Sarid. Exploring Scanning Probe Microscopy with MATHEMATICA. Second edition. (WILEY-VCH Verlag GmbH \& Co. KGaA, Weinheim, 2007)

[17] M.A. Lantz, S.J. O’Shea, A.C.F. Hoole, M.E. Welland. Appl. Phys. Lett., 70 (8), 970 (1997). DOI: https://doi.org/10.1063/1.118476

[18] А.В. Анкудинов, М.М. Халисов. ЖТФ, 90 (11), 1951 (2020). DOI: 10.21883/JTF.2020.11.49989.117-20

[19] Электронный ресурс. Режим доступа: https://www.ntmdtsi.ru/ resources/spm-theory/theoretical-background-of-spm

[20] J.E. Sader, J.W.M. Chon, P. Mulvaney. Rev. Sci. Instrum., 70 (10), 3967 (1999). DOI: https://doi.org/10.1063/1.1150021

[21] В.Л. Миронов. Основы сканирующей зондовой микроскопии: учебное пособие для студентов старших курсов вузов (Техносфера, М., 2009) 